\title{
The Atacama Cosmology Telescope: Probing the baryon content of SDSS DR15 galaxies with the thermal and kinematic Sunyaev-Zel'dovich effects
}

E. M. Vavagiakis, ${ }^{1}$ P. A. Gallardo, ${ }^{1}$ V. Calafut,${ }^{2}$ S. Amodeo, ${ }^{2}$ S. Aiola, ${ }^{3}$ J. E. Austermann, ${ }^{4}$ N. Battaglia, ${ }^{2}$ E. S. Battistelli, ${ }^{5}$ J. A. Beall, ${ }^{4}$ R. Bean, ${ }^{2}$ J. R. Bond, ${ }^{6}$ E. Calabrese, ${ }^{7}$ S. K. Choi, ${ }^{1,2}$ N. F. Cothard,${ }^{8}$ M. J. Devlin, ${ }^{9}$ C. J. Duell, ${ }^{1}$ S. M. Duff, ${ }^{4}$ A. J. Duivenvoorden, ${ }^{10}$ J. Dunkley, ${ }^{10,11}$ R. Dunner, ${ }^{12}$ S. Ferraro, ${ }^{13,14}$ Y. Guan, ${ }^{15}$ J. C. Hill, ${ }^{3,16}$ G. C. Hilton, ${ }^{4}$ M. Hilton, ${ }^{17}$

R. Hložek, ${ }^{18,19}$ Z. B. Huber, ${ }^{1}$ J. Hubmayr, ${ }^{4}$ K. M. Huffenberger, ${ }^{20}$ J. P. Hughes, ${ }^{21}$ B. J. Koopman, ${ }^{22}$ A. Kosowsky, ${ }^{15}$

Y. Li, ${ }^{1}$ M. Lokken, ${ }^{6,18,19}$ M. Madhavacheril, ${ }^{23}$ J. McMahon, ${ }^{24,25,26,27}$ K. Moodley, ${ }^{17,28}$ S. Naess, ${ }^{3}$ F. Nati, ${ }^{29}$

L. B. Newburgh, ${ }^{22}$ M. D. Niemack,${ }^{1,2}$ L. A. Page, ${ }^{10}$ B. Partridge, ${ }^{30}$ E. Schaan, ${ }^{12,13}$ A. Schillaci, ${ }^{31}$ C. Sifón, ${ }^{32}$

D. N. Spergel, ${ }^{3,11}$ S. T. Staggs, ${ }^{10}$ J. N. Ullom, ${ }^{4}$ L. R. Vale, ${ }^{4}$ A. Van Engelen, ${ }^{33}$ J. Van Lanen, ${ }^{4}$ E. J. Wollack, ${ }^{34}$ and Z. Xu ${ }^{9,35}$

${ }^{1}$ Department of Physics, Cornell University, Ithaca, New York 14853, USA

${ }^{2}$ Department of Astronomy, Cornell University, Ithaca, New York 14853, USA

${ }^{3}$ Center for Computational Astrophysics, Flatiron Institute, New York, New York 10010, USA

${ }^{4}$ NIST Quantum Sensors Group, 325 Broadway, Boulder, Colorado 80305, USA

${ }^{5}$ Physics Department, Sapienza University of Rome, Piazzale Aldo Moro 5, I-00185 Rome, Italy

${ }^{6}$ Canadian Institute for Theoretical Astrophysics, University of Toronto, Toronto, Ontario M5S 3H8, Canada

${ }^{7}$ School of Physics and Astronomy, Cardiff University, The Parade, Cardiff CF24 3AA, United Kingdom

${ }^{8}$ Department of Applied and Engineering Physics, Cornell University, Ithaca, New York 14853, USA

${ }^{9}$ Department of Physics and Astronomy, University of Pennsylvania, 209 South 33rd Street, Philadelphia, Pennsylvania 19104, USA

${ }^{10}$ Joseph Henry Laboratories of Physics, Jadwin Hall, Princeton University, Princeton, New Jersey 08544, USA

${ }^{11}$ Department of Astrophysical Sciences, Peyton Hall, Princeton University, Princeton, New Jersey 08544, USA

${ }^{12}$ Instituto de Astrofísica and Centro de Astro-Ingeniería, Facultad de Física, Pontificia Universidad Católica de Chile, Avenida Vicuña Mackenna 4860, 7820436, Macul, Santiago, Chile

${ }^{13}$ Lawrence Berkeley National Laboratory, One Cyclotron Road, Berkeley, California 94720, USA

${ }^{14}$ Berkeley Center for Cosmological Physics, UC Berkeley, California 94720, USA

${ }^{15}$ Department of Physics and Astronomy, University of Pittsburgh, Pittsburgh, Pennsylvania 15260, USA

${ }^{16}$ Department of Physics, Columbia University, New York, New York 10027, USA

${ }^{17}$ Astrophysics Research Centre, University of KwaZulu-Natal, Westville Campus, Durban 4041, South Africa

${ }^{18}$ David A. Dunlap Department of Astronomy and Astrophysics, University of Toronto, 50 St. George Street, Toronto, Ontario M5S 3H4, Canada

${ }^{19}$ Dunlap Institute for Astronomy and Astrophysics, University of Toronto, 50 St. George Street, Toronto, Ontario M5S 3H4, Canada

${ }^{20}$ Department of Physics, Florida State University, Tallahassee, Florida 32306, USA

${ }^{21}$ Department of Physics and Astronomy, Rutgers, The State University of New Jersey, 136 Frelinghuysen Road, Piscataway, New Jersey 08854-8019, USA

${ }^{22}$ Department of Physics, Yale University, New Haven, Connecticut 06511, USA

${ }^{23}$ Centre for the Universe, Perimeter Institute for Theoretical Physics, Waterloo, Ontario N2L 2Y5, Canada

${ }^{24}$ Department of Physics, University of Chicago, Chicago, Illinois 60637, USA

${ }^{25}$ Department of Astronomy and Astrophysics, University of Chicago, 5640 South Ellis Avenue, Chicago, Illinois 60637, USA

${ }^{26}$ Kavli Institute for Cosmological Physics, University of Chicago, 5640 South Ellis Avenue, Chicago, Illinois 60637, USA

${ }^{27}$ Enrico Fermi Institute, University of Chicago, Chicago, Illinois 60637, USA

${ }^{28}$ School of Mathematics, Statistics and Computer Science, University of KwaZulu-Natal, Westville Campus, Durban 4041, South Africa

${ }^{29}$ Department of Physics, University of Milano-Bicocca, Piazza della Scienza 3, 20126 Milano (MI), Italy

${ }^{30}$ Department of Physics and Astronomy, Haverford College, Haverford, Pennsylvania 19041, USA

${ }^{31}$ Department of Physics, California Institute of Technology, Pasadena, California 91125, USA

${ }^{32}$ Instituto de Física, Pontificia Universidad Católica de Valparaíso, Casilla 4059, Valparaíso, Chile

${ }^{33}$ School of Earth and Space Exploration, Arizona State University, Tempe, Arizona 85287, USA 


\author{
${ }^{34}$ NASA Goddard Space Flight Center, Greenbelt, Maryland 20771, USA \\ ${ }^{35}$ MIT Kavli Institute, Massachusetts Institute of Technology, Cambridge, Massachusetts 02139, USA
}

(Received 4 February 2021; accepted 27 May 2021; published 6 August 2021)

We present measurements of the average thermal Sunyaev Zel'dovich (tSZ) effect from optically selected galaxy groups and clusters at high signal-to-noise (up to 12 $\sigma$ ) and estimate their baryon content within a 2.1' radius aperture. Sources from the Sloan Digital Sky Survey Baryon Oscillation Spectroscopic Survey DR15 catalog overlap with 3,700 sq deg of sky observed by the Atacama Cosmology Telescope (ACT) from 2008 to 2018 at 150 and $98 \mathrm{GHz}$ (ACT DR5), and 2,089 sq deg of internal linear combination component-separated maps combining ACT and Planck data (ACT DR4). The corresponding optical depths $\bar{\tau}$, which depend on the baryon content of the halos, are estimated using results from cosmological hydrodynamic simulations assuming an active galactic nuclei feedback radiative cooling model. We estimate the mean mass of the halos in multiple luminosity bins, and compare the tSZ-based $\bar{\tau}$ estimates to theoretical predictions of the baryon content for a Navarro-Frenk-White profile. We do the same for $\bar{\tau}$ estimates extracted from fits to pairwise baryon momentum measurements of the kinematic SunyaevZel'dovich effect (kSZ) for the same dataset obtained in a companion paper. We find that the $\bar{\tau}$ estimates from the tSZ measurements in this work and the kSZ measurements in the companion paper agree within $1 \sigma$ for two out of the three disjoint luminosity bins studied, while they differ by $2-3 \sigma$ in the highest luminosity bin. The optical depth estimates account for one-third to all of the theoretically predicted baryon content in the halos across luminosity bins. Potential systematic uncertainties are discussed. The tSZ and $\mathrm{kSZ}$ measurements provide a step toward empirical Compton- $\bar{y}-\bar{\tau}$ relationships to provide new tests of cluster formation and evolution models.

DOI: 10.1103/PhysRevD.104.043503

\section{INTRODUCTION}

The thermal Sunyaev-Zel'dovich (tSZ) effect is a consequence of cosmic microwave background (CMB) photons inverse-Compton scattering off electrons in hot, ionized gas, especially that in the intracluster medium (ICM) of galaxy clusters and groups, resulting in a shift in the CMB blackbody spectrum [1,2]. An SZ effect arising from the motion of these groups and clusters, the kinematic SZ (kSZ) effect, has a different spectral signature and is an order of magnitude smaller in amplitude than the tSZ signal, making it significantly more difficult to detect in maps of the CMB. Together, the SZ effects encode rich information about galaxy groups.

The distortion in the CMB due to the tSZ effect depends on the optical depth of the gas $\bar{\tau}$ as well as the electron temperature $T_{e}$ and is proportional to the Compton- $y$ parameter. In its sensitivity to the cluster's integrated line-of-sight pressure profile, the tSZ effect is a valuable, largely redshift-independent probe of gas in the ICM. Measurements of the tSZ effect allow us to study the thermodynamics of the cluster gas, including processes such as active galactic nuclei (AGN) feedback, star formation, radiative cooling, and cluster merger histories. The tSZ effect can also give us information about the shapes and extents of cluster gravitational potential wells and dark matter halos $[3,4]$. By tracing the electron distribution within groups and clusters, the tSZ effect is sensitive to the poorly understood spatial distribution of ionized gas and the baryon content. We are able to estimate the optical depth of the gas within halos by combining tSZ measurements with cosmological hydrodynamic simulations [3]. In the case of the "missing baryon" problem, observations suggest that this gas contains fewer baryons than would be predicted by massdensity profile models neglecting heating processes [5-9]. These baryons are thought to be located at the outskirts of groups in the diffuse warm-hot intergalactic medium, where they are not easily measured by $\mathrm{x}$-ray observations or the tSZ effect [5,10-14].

While the kSZ effect has an amplitude proportional to both the total cluster gas mass and the cluster's line-of-sight velocity, it is independent of the gas temperature. When both the tSZ and kSZ effects are measured for the same sample of sources and the optical depth (and thus the total cluster gas mass) is modeled from the tSZ data, the combination can be used to convert the pairwise momentum [15] measured from the kSZ effect into pairwise velocity, which in turn can be used to constrain cosmological parameters, such as the sum of the neutrino masses $[16,17]$.

To use tSZ measurements to probe cluster properties, we must extract the tSZ signal from the microwave sky at high significance. Cleaning the tSZ signal from sources of contamination such as competing astrophysical and cosmological signals, instrumentation and atmospheric noise, and dusty galaxy and synchrotron emission can be a challenge. Recent efforts by the Atacama Cosmology Telescope (ACT) [18-21], South Pole Telescope (SPT) $[22,23]$, and Planck Collaborations [24] have utilized wide- 

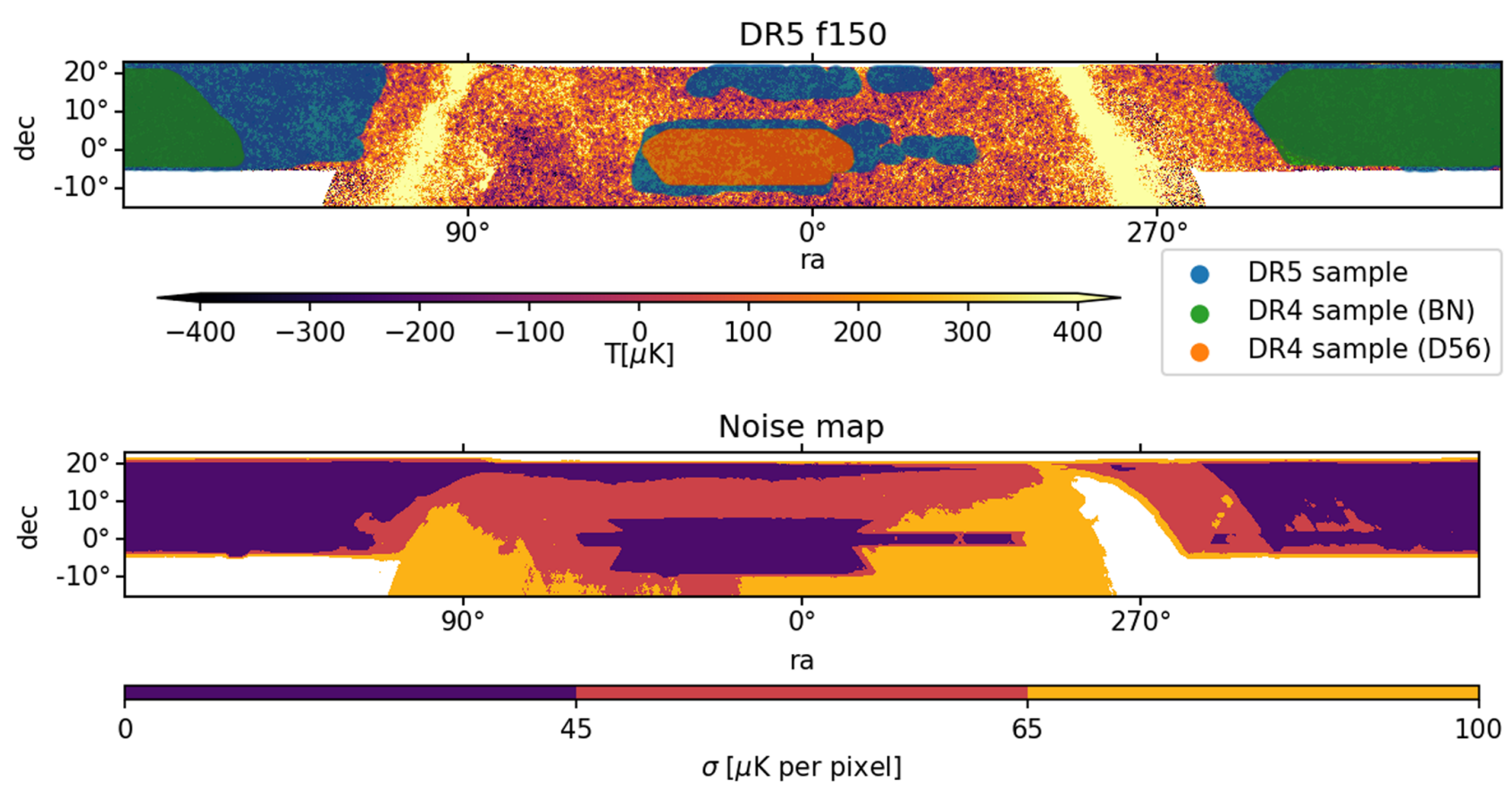

FIG. 1. Top: The ACT + Planck map used for the DR5 f150 analysis with the overlapping 343,647 SDSS DR15 selected sources plotted in blue over 3,700 sq deg, and the BN and D56 areas covered by the ILC maps plotted in green and orange, respectively. Bottom: The inverse white noise variance map associated with the DR5 f150 coadded ACT + Planck map highlighting regions representing a noise equivalence of 45 and $65 \mu \mathrm{K}$ per pixel (with a 0.5 arcmin resolution plate Carré projection), which were used to cut the SDSS sample for the DR5 f150 analysis. The orange and yellow regions of higher noise overlapped with $27 \%$ of the DR 15 sample. Results are shown for the more conservative $45 \mu \mathrm{K}$ per pixel inverse white noise variance map cut shown in purple. We performed an equivalent cut for the DR5 f090 map and analysis.

field multifrequency data to produce measurements of the tSZ effect in CMB data. Heritage for both tSZ stacking and multifrequency dust reconstruction in ACT data includes multiple recent publications [25-27].

In this work we use multifrequency CMB temperature maps [28] (Fig. 1) from ACT (Data Release 5, DR5) and Planck data, along with component-separated Compton-y maps [29] (DR4) to extract our tSZ signals. We perform aperture photometry $(\mathrm{AP})$ on stacked $18^{\prime} \times 18^{\prime}$ map cutouts (submaps) centered on sources from the Sloan Digital Sky Survey (SDSS) Baryon Oscillation Spectroscopic Survey (BOSS DR15 [30]) in luminosity-selected bins. We bin by luminosity to target the highest signal-to-noise SZ measurements and enable comparisons between independent bins of sources with differing average masses. We select 343,647 luminous red galaxies (LRGs) from the DR15 catalog based on an inverse white noise variance map cut, point source mask, and Planck Galactic plane mask used in the production of the 2015 Planck Compton-y map [31]. We then clean the tSZ signals by removing contaminating emission from dusty galaxies. We convert these tSZ signals to estimates of optical depth $\bar{\tau}$ by using a hydrodynamical model [3].

In a companion paper, Calafut et al. 2020 (henceforth C21) [32], we report a measurement of the pairwise kSZ signal using the same datasets described here to measure the kSZ effect at $>5 \sigma$, a significant improvement over our previous results (De Bernardis et al. 2017, henceforth DB17 [33]). Previous measurements using the pairwise estimator [34] have been reported by the Planck Collaboration using galaxies from SDSS [35], and the South Pole Telescope Collaboration using galaxies from the Dark Energy Survey [36]. In C21, we obtain optical depth estimates by fitting to an analytical kSZ signal model. In this work, we compare these estimates to $\bar{\tau}$ estimates obtained through tSZ measurements.

In 2011, Hand et al. presented a measurement of the tSZ effect in ACT maps using a matched-filtering method with an assumed profile [37]. In DB17, a $600 \mathrm{deg}^{2}$ map from ACT Data Release 3 (DR3) was analyzed in combination with sources from the SDSS DR11 galaxy catalog (Fig. 2) also using a matched filter for the tSZ signal extraction. Then $\bar{\tau}$ was estimated from the same hydrodynamical simulations used in this work. In this work, we adopt the aperture photometry approach for both the tSZ and kSZ analysis and estimate both the tSZ uncertainties and kSZ covariance matrix using jackknife estimates for consistency. The tSZ results presented here are consistent with those in DB17, with smaller uncertainties. The details of the kSZ analysis that differ between this work and DB17 are discussed in C21. Although our galaxy samples are different from those in Hand et al. [37], our results are 


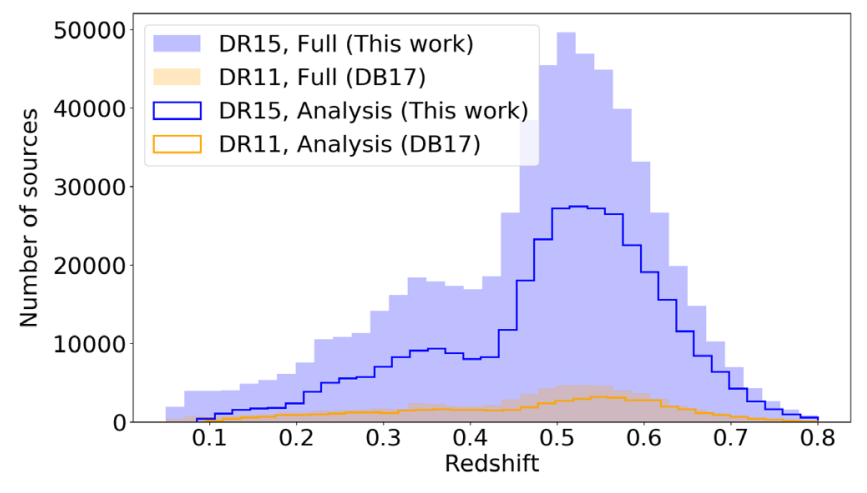

FIG. 2. The SDSS DR15 redshift distribution for the 602,461 total galaxy sample and selected 343,647 galaxy sample for analysis overlapping with the ACT + Planck DR5 map, as compared to the $\sim 9$ and $\sim 7$ times fewer DR11 galaxies overlapping with the ACT DR3 area and those used for the 2017 result (DB17), respectively.

statistically consistent with those results for bins with similar average luminosity.

A pair of contemporary papers from ACT, Amodeo et al. 2020 (A20) [38] and Schaan et al. 2020 (S20) [39], use the same ACT DR5 maps [28] or component-separated maps [29] as are used here and in C21, but with a different galaxy sample, to focus on the radial dependence of the kSZ and tSZ signals, i.e., on the baryon profiles. Rather than the pairwise estimator used in $\mathrm{C} 21, \mathrm{~S} 20$ uses the velocity reconstruction $\mathrm{kSZ}$ estimator, which is convenient for measuring the baryon density profile. To obtain a complete picture of the gas thermodynamics, A20 and S20 stack on the CMASS and LOWZ galaxy samples [40], for which clustering and galaxy lensing measurements are available. Because the galaxy samples are different in this work from A20 and S20, and have different host halo masses, the results from these papers are not directly comparable to ours; however the rough signal-to-noise ratios obtained are comparable. The promise of the pairwise kSZ estimator used in C21 lies in its potential for measurements of the pairwise velocity, if the optical depths of the sources are known. In this paper, we investigate methods of constraining the optical depth. Overall, these various papers are complementary, and highlight the wealth of information in joint $\mathrm{kSZ}$ and $\mathrm{tSZ}$ measurements. In Sec. II we describe the ACT + Planck maps we analyze, and discuss the selection and binning of the SDSS data used for this work. In Sec. III we summarize our aperture photometry filtering approach toward extracting tSZ signals from our maps and measuring the average Compton- $y$ within our $2.1^{\prime}$ aperture, $\bar{y}$. We describe how we use a scaling relation from hydrodynamic simulations to convert these measurements of $\bar{y}$ into estimates of halo optical depth, $\bar{\tau}$. We explore dust and beam corrections and possible systematic effects in Sec. III C. We calculate theoretical estimates for these halos in Sec. III E in order to compare with our estimates. In C21, kSZ-based estimates of $\bar{\tau}$ are obtained for the same dataset. We discuss this in Sec. III D. We present the tSZ $\bar{y}$ measurements for each bin and estimated $\bar{\tau}$ values from the tSZ and kSZ results in Sec. IV. The optical depth estimates from the kSZ signals from C21 and tSZ signals from this work are compared with the theoretical estimates and with one another in Sec. IV C. We assume the Planck cosmology for a flat universe [31]: $\Omega_{b} h^{2}=0.02225, \quad \Omega_{c} h^{2}=0.1198, \quad H_{0}=67.3 \mathrm{~km} / \mathrm{s} / \mathrm{Mpc}$, $\sigma_{8}=0.83, n_{s}=0.964$. Combining the SZ measurements provides a step toward using observations to refine our models of galaxy formation and feedback, leading toward an empirical $\bar{y}-\bar{\tau}$ relationship free of the assumptions required in current simulations.

\section{DATA}

\section{A. ACT data}

This analysis uses a component-separated internal linear combination (ILC) Compton- $y$ map referred to as the DR4 ILC map covering the BN (1,633 sq deg) and D56 (456 sq deg) regions (see Fig. 1). The ILC map is comprised of two seasons of observations with the ACTPol receiver [41] (ACT DR4) [29]. In C21, the DR4 ILC map used for the $\mathrm{kSZ}$ analysis is a CMB $+\mathrm{kSZ}$ map. The ILC approach was designed to account for the anisotropic noise found in ground-based CMB experiments. The ILC method combines multifrequency data from Planck and ACT and constructs wide-area, arcminute-resolution component-separated maps of CMB temperature and the tSZ effect. Cosmic infrared background-deprojected Compton- $y$ maps are also available, but were not analyzed in this work due to their higher noise. Additionally, ACT DR5 single frequency data at 150 and $98 \mathrm{GHz}$ are coadded with data from Planck at 100 and $143 \mathrm{GHz}$ to cover about 21,100 sq deg of sky $(3,700 \mathrm{sq}$ deg of which overlaps with the SDSS data sample) [28]. These maps are referred to as the DR5 f150 and DR5 f090 maps. While the DR5 data have the highest signal-to-noise ratio, the DR4 ILC results serve to check the consistency with a map that already combines the multifrequency information. A higher signal-to-noise ILC map for the DR5 data is not yet available and so was not analyzed here, but will be useful for future measurements. The beams have FWHM $=1.3^{\prime}, 2.1^{\prime}$, and $1.6^{\prime}$ for the DR5 f150, DR5 f090, and DR4 ILC maps, respectively, with associated uncertainties of several percent [28].

The inverse white noise variance map associated with the DR5 data is used for cutting the SDSS data sample (Sec. II B) and for weighting (Sec. III B). For the ILC analysis, the DR5 f090 inverse white noise variance map is used for weighting as it best estimates the noise properties in the ILC map. Figure 1 shows the ACT + Planck map overlaid with the selected 343,647 sources from the SDSS DR15 catalog.

For the tSZ measurements, submaps are stacked on the locations of galaxies from the public Sloan Digital Sky 
TABLE I. Luminosity bin labels (the * bins are also analyzed in C21) and cuts (Appendix B) equivalent halo mass cuts, average stellar mass per bin, number of sources $(\mathrm{N})$, average luminosity $(\langle L\rangle)$, and average redshift $(\langle z\rangle)$ per luminosity bin for the final DR15 samples used in the DR5 f150, DR5 f090, and ILC Compton- $y$ map analyses. These samples have the noise cut, point source masks, and Galactic plane mask applied. The samples differ between the coadded and ILC maps due to the difference in footprints of the two maps, with the ILC maps covering a smaller area on the sky.

\begin{tabular}{|c|c|c|c|c|c|c|c|c|c|}
\hline \multirow[b]{2}{*}{ Bin } & \multirow[b]{2}{*}{ Luminosity cut $/ 10^{10} L_{\odot}$} & \multirow[b]{2}{*}{$M_{\text {vir }}$ cut $/ 10^{13} M_{\odot}$} & \multirow[b]{2}{*}{$\left\langle M_{*}\right\rangle / 10^{11} M_{\odot}$} & \multicolumn{3}{|c|}{ DR5 f150, DR5 f090 } & \multicolumn{3}{|c|}{ DR4 ILC } \\
\hline & & & & $\mathrm{N}$ & $\langle L\rangle / 100^{10} L_{\odot}$ & $\langle z\rangle$ & $\mathrm{N}$ & $\langle L\rangle / 10^{10} L_{\odot}$ & $\langle z\rangle$ \\
\hline L43* & $L>4.30$ & $M>0.52$ & 2.21 & 343,647 & 7.4 & 0.49 & 190,551 & 7.4 & 0.50 \\
\hline L61* & $L>6.10$ & $M>1.00$ & 2.61 & 213,070 & 8.7 & 0.51 & 118,852 & 8.7 & 0.51 \\
\hline L79* & $L>7.90$ & $M>1.66$ & 3.17 & 103,159 & 10.6 & 0.53 & 57,828 & 10.6 & 0.54 \\
\hline L98 & $L>9.80$ & $M>2.59$ & 3.84 & 46,956 & 12.8 & 0.56 & 26,308 & 12.8 & 0.57 \\
\hline L116 & $L>11.60$ & $M>3.70$ & 4.50 & 23,504 & 15.0 & 0.58 & 13,277 & 15.0 & 0.59 \\
\hline $\mathrm{L}^{4} 3 \mathrm{D}^{*}$ & $4.30<L<6.10$ & $0.52<M<1.00$ & 1.57 & 130,577 & 5.2 & 0.48 & 71,699 & 5.2 & 0.48 \\
\hline L61D* & $6.10<L<7.90$ & $1.00<M<1.66$ & 2.08 & 109,911 & 6.9 & 0.48 & 61,024 & 6.9 & 0.48 \\
\hline L79D & $7.90<L<9.80$ & $1.66<M<2.59$ & 2.61 & 56,203 & 8.7 & 0.51 & 31,520 & 8.7 & 0.52 \\
\hline L98D & $9.8<L<11.60$ & $2.59<M<3.70$ & 3.18 & 23,452 & 10.6 & 0.54 & 13,031 & 10.6 & 0.55 \\
\hline
\end{tabular}

Survey Large Scale Structure DR15 catalog [42] from the BOSS survey [43]. The tSZ signal from the CMB map is measured at the positions of these objects. We assume that higher luminosity optical galaxies trace more massive halos. Systematic effects associated with this assumption are discussed in the context of these data in C21, and have been previously explored for precursor data in [44]. The assumption that each galaxy traces one halo is discussed in Sec. III C.

\section{B. SDSS data}

The 602,461 DR15 LRGs overlapping with the DR5 f150 ACT + Planck map were selected for use in this analysis through a luminosity cut, a cut based on the CMB map noise level, point source masks, and a Galactic plane mask. The catalog was downloaded from the SDSS SkyServer using the query presented in Appendix A. The full catalog, with flags for the cuts described below, is publicly available [45]. The luminosities of the sources are calculated based on their (multiband) dereddened SDSS composite model magnitudes and $\mathrm{K}$-corrected using the k_correct [46,47] software according to the luptitude to flux conversion outlined in [48]. The DR15 luminosities of the selected sources range from $4.30 \times 10^{10} L_{\odot}$ to $2.61 \times$ $10^{12} L_{\odot}$ with an average luminosity of $7.38 \times 10^{10} L_{\odot}$. Luminosity bins for joint tSZ and kSZ analyses with C21 were chosen to match two of the luminosity cuts from DB17 $\left(L=7.9 \times 10^{10} L_{\odot}\right.$ and $\left.L=6.1 \times 10^{10} L_{\odot}\right)$ as well as one lower luminosity cut $\left(L=4.3 \times 10^{10} L_{\odot}\right)$. The three disjoint bins based on these cuts were selected to have roughly equal spacing, and such that each bin has over 100,000 galaxies that pass cuts for analysis with the DR5 maps (Table I). The cumulative luminosity bins include the highest signal-to-noise SZ measurements, while the disjoint bins enable comparisons between independent bins. Since the tSZ signal-to-noise ratio is higher for high mass halos, we also perform the tSZ analysis for the two highest mass bins from DB17 (L98 and L116) which are not studied in C21. A plot of the luminosity distribution and bins is shown in Appendix B.

After we selected luminosity bins and applied the minimum luminosity cut, which removed 80,162 lower luminosity sources from the sample, we performed an inverse white noise variance cut. Figure 1 shows the inverse white noise variance map that was used to explore cuts in the DR15 sample. Initially two different cuts were studied (45 and $65 \mu \mathrm{K}$ per pixel). The more conservative cut of $45 \mu \mathrm{K}$ per pixel was selected for all subsequent analyses based on a signal-blind uncertainty analysis which compared the jackknife error bars on the AP analysis for the samples cut by $45 \mu \mathrm{K}$ per pixel, $65 \mu \mathrm{K}$ per pixel, and no noise cut. The $45 \mu \mathrm{K}$ per pixel cut removes $27 \%$, or 140,209 sources from the overlapping sample and improves the jackknife error bars as compared to no noise cut.

Galactic plane masking was then performed with the mask used in the production of the 2015 Planck Compton- $y$ map to reduce Galactic contamination [31]. The 50\% mask was selected to conservatively cut sources from the Galactic plane region of the ACT + Planck map, resulting in a cut of 26,521 additional sources from the DR15 sample.

To mask point sources we first used the two source masks developed for Choi et al. [49]. For the lowest noise D56 region a $15 \mathrm{mJy}$ point source mask was applied, and for the higher noise regions a $100 \mathrm{mJy}$ mask was applied. Together these masks removed an additional 11,922 sources from our sample, leaving us with a final selected sample of 343,647 sources after all masks and cuts are applied.

Figure 2 shows the redshift distributions of the 343,647 DR15 galaxies overlapping with the ACT + Planck map after the luminosity, inverse white noise variance map, Galactic plane mask, and point source mask cuts. The 
67,938 DR11 sources overlapping with the coadded ACT DR3 map used in our 2017 result are also shown for comparison (DB17 [33]). The redshifts range from 0.08 to 0.8 with an average reshift of 0.49 for the DR 15 catalog.

Histograms of the luminosities depicting cuts and bins are provided in Appendix B. The properties of each luminosity-selected bin are summarized in Table I. The mean stellar masses are estimated from the mean luminosities assuming $M_{*} / L=3.0$ as predicted by the Chabrier IMF [50] and discussed in [51-54]. The mean halo masses are derived from the $M_{*}-M_{\text {vir }}$ relation from abundance matching as described in [51]. Abundance matching is a statistical technique to model the correlations between galaxy and halo properties based on mapping galaxies to dark matter halos of the same number density in the Universe.

The same mass cuts and average redshifts of the sources reported in Table I are used when calculating the linear model prediction from the $\mathrm{kSZ}$ measurements in $\mathrm{C} 21$.

\section{ANALYSIS}

We use AP to filter the Compton- $y$ and DR5 f150 maps and extract the tSZ signals by stacking on source-centered submaps. The same 2.1' AP filter is used in the kSZ analysis in $\mathrm{C} 21$. This approach to comparing estimated optical depths is an improvement over the 2017 result in which a matched filter was used for the tSZ analysis while AP was used for the kSZ analysis.

\section{A. Filtering CMB maps}

We select submaps of $18^{\prime} \times 18^{\prime}$ (about 3 times larger than the outer diameter of the AP annulus) of pixel size $0.5^{\prime}$, interpolate in the Fourier domain with a pixel size of $0.1^{\prime}$ per pixel, and reproject to a coordinate system centered at the galaxy center position using PIXELL [55]. A comparison between different pixelization approaches is discussed in C21 Appendix A, and we use here the same approach adopted in C21. On each source-centered submap, we draw an aperture of $R_{1}=2.1^{\prime}$ located at the central coordinate in RA and DEC provided by the DR15 catalog. The signal associated with the sources is taken to be the average within this aperture, minus the average of the pixels within an annulus of inner radius $R_{1}=2.1^{\prime}$ and an outer radius of $\sqrt{2} R_{1}$. The $2.1^{\prime}$ size of this annulus was selected to correspond to $\sim 0.8 \mathrm{Mpc}$ based on the average angular diameter distance for the source sample and assumed cosmology (Sec. I). An associated weight for each source is assigned by taking the average within an $R_{1}=2.1^{\prime}$ disk centered on the same source position on an $18^{\prime} \times 18^{\prime}$ submap taken from the inverse white noise variance map. This value is used for weighting the signals in the tSZ analysis.

\section{B. tSZ signal extraction}

We stack on the positions of DR15 sources in the nine luminosity bins listed in Table I to measure the average $\delta T_{\text {tSZ }}$ in differential CMB temperature units from the DR5 f090 and DR5 f150 maps, and the average Compton- $y$ for the DR4 ILC map. Round number luminosity cuts were chosen to yield five cumulative and disjoint luminosity bins, before any cuts were made to the DR 15 sample, or any analyses were run. The weighted averages of the stacked submaps for each bin are shown in Fig. 3, and the radial averages of these submaps are plotted in Fig. 4. The signal associated with each source in a bin is taken to be the average of the $\langle$ disk $\rangle-\langle$ ring $\rangle$ values per source, weighted by the associated average inverse variance weight per source. The presence of dust emission on angular scales comparable to or less than the beam size is visible in all the maps analyzed, but is most prominent in the DR5 f150 map (Figs. 3 and 4). We study the impact of discarding the pixels within the beam radius from our analysis, as discussed further in Sec. III C. This core-excised AP method removes the SZ signal within the beam radius along with the dust emission; it has a small (generally $<1 \sigma$ ) effect on the tSZ signals (Fig. 5), so it is not adopted for the final analysis. The $2.1^{\prime}$ AP signals are then averaged in each bin to obtain a stacked tSZ signal, $\delta T_{\text {tSZ }}$. The uncertainty associated with the stacked tSZ signal for each bin is obtained using a jackknife estimation method over the sources with 2,000 iterations per bin.

We correct the $\delta T_{\mathrm{tSZ}}$ and $\bar{y}$ estimates obtained from the DR5 f090 map to account for the larger DR5 f090 beam $\left(\mathrm{FWHM}=2.1^{\prime}\right)$ compared to the DR5 f150 map $\left(\mathrm{FWHM}=1.3^{\prime}\right)$ [28]. We also correct the $\bar{y}$ estimates from the DR4 ILC map which has an effective beam corresponding to a 1.6 FWHM Gaussian [29]. We compute the beam correction factors as follows: We consider a fiducial pressure profile for the average virial mass $\left(M_{\mathrm{vir}}\right)$ in each bin [56]; we derive three estimates of the Compton- $y$ signal in a 2.1' AP filter, convolved with the $\mathrm{f} 150$ and f090 beams from [28] and with a 1.6' FWHM Gaussian beam, using Mop-c GT [57] (see [38] for a detailed description on how the projection from pressure to Compton- $y$ profile and the beam convolution are implemented). We find an AP beam correction of $31 \%$ for the DR5 f090 measurements, and a beam correction of $-5 \%$ for the DR4 ILC measurements. Thus, the DR5 f090 $\delta T_{\text {tSZ }}$ measurements are multiplied by 1.3, which propagates into the DR5 f090 Compton-y estimates and resulting analyses, and the DR4 ILC $\bar{y}$ measurements are multiplied by 0.95 . The value for the correction changes negligibly across luminosity bins, and is insensitive to our estimate of $M_{\text {vir }}$ (the change in factor is $\sim 1 \%$ if we vary $M_{\text {vir }}$ in our mass range). While Figs. 3 and 4 show the raw data, the DR5 f090 and DR4 ILC data in the rest of this work are multiplied by these factors. We note that because the $\mathrm{f} 150$ beam is not exactly Gaussian, but features secondary lobes, we get a negative $(<1)$ ILC correction, 

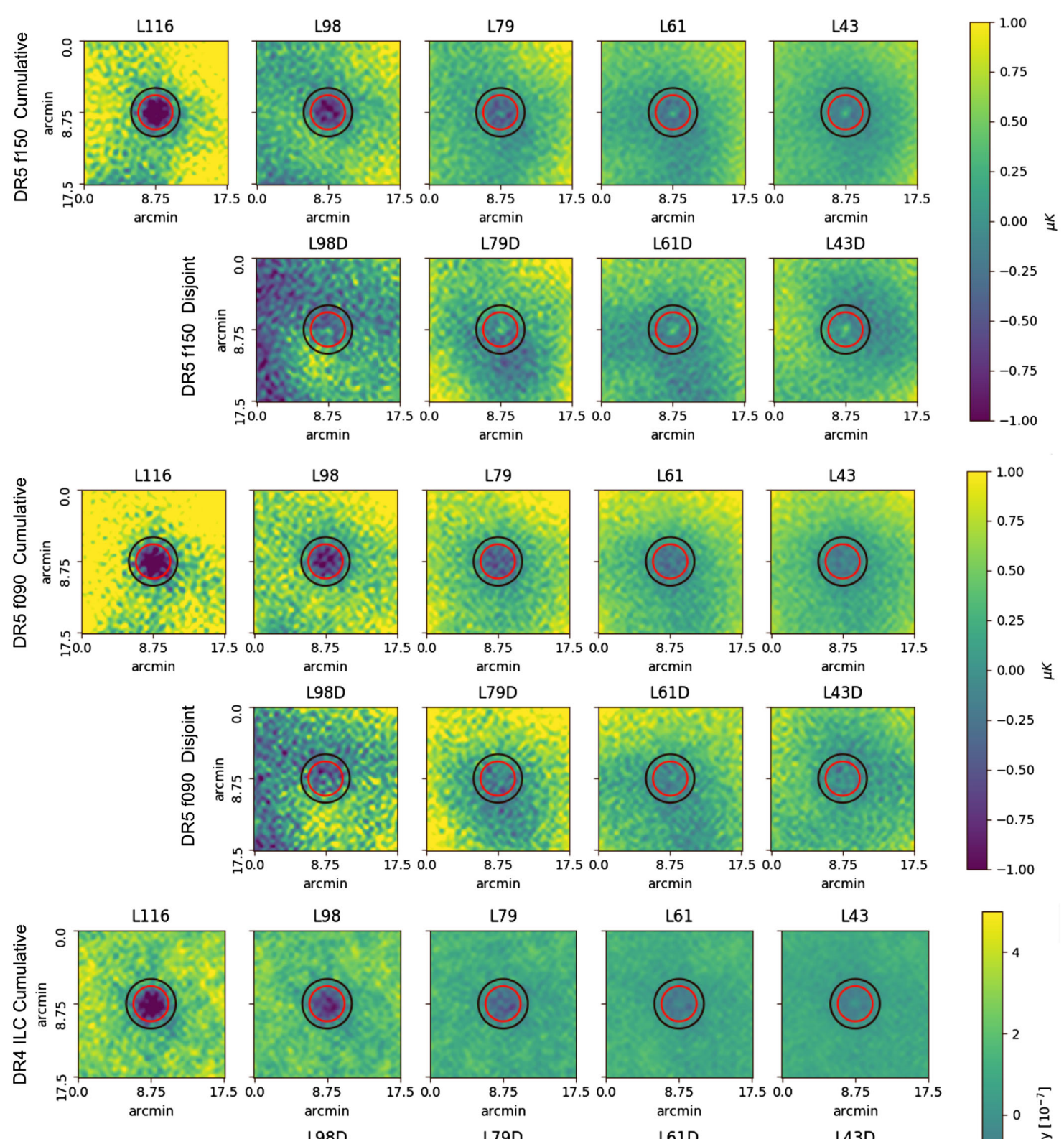

179

L61
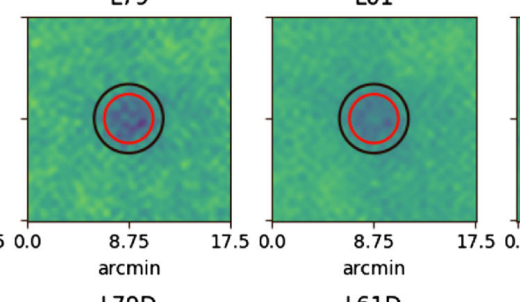

L43
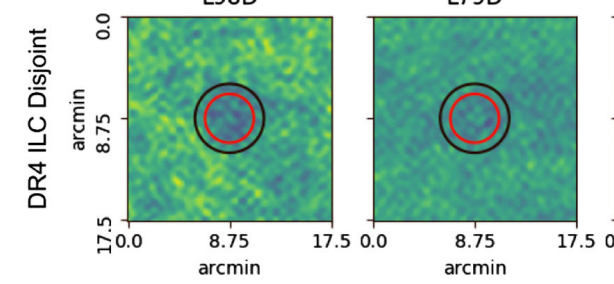

L61D
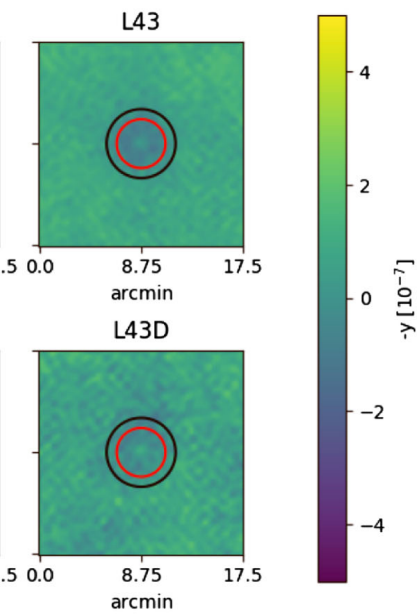

FIG. 3. Stacked raw submaps for the five cumulative (top) and four disjoint (bottom) luminosity bins as defined in Table I, for the DR5 f150 map (top two rows, in differential units of CMB temperature which are calibrated to Planck [28]), the DR5 f090 map (middle two rows, in the same units as the top two rows), and the DR4 ILC Compton- $y$ maps (bottom two rows, in negative units of $y$ to better compare to the coadded maps). The submaps represent the weighted average submaps of the sources in a given bin, where the weight for each source is taken to be the average value inside the accompanying $R_{1}=2.1$ submap in the inverse white noise variance map. The sub$0.5^{\prime}$-scale structure in the submaps is an artifact of the subpixel interpolation and is not physical. The maps are normalized with the average value within the AP annulus, such that the mean of the pixels in the annulus in these maps is equal to zero. The apertures used for the tSZ and kSZ AP are drawn, where $R_{1}=2 \cdot 1^{\prime}$ (red) and $\sqrt{2} R_{1}$ (black). Radial averages of these submaps are plotted in Fig. 4. A central bright spot due to dust on approximately the beam scale can be seen across luminosity bins in the DR5 f150 submaps, but not the DR5 f090 submaps. Dust contamination of the DR4 ILC maps is more subtle, but can be noticed in radial average plots (Fig. 4). 

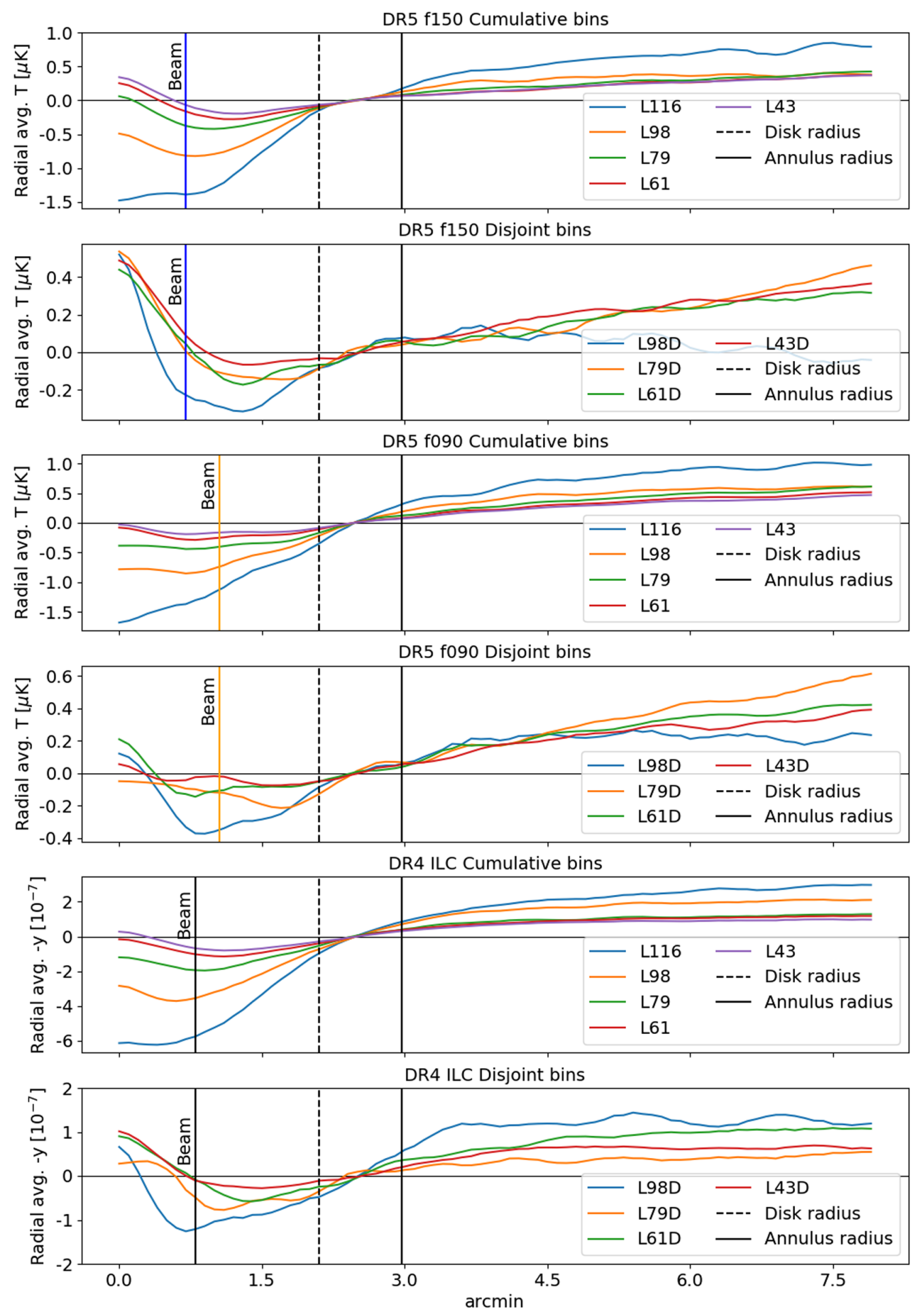

FIG. 4. Radial average of the stacked submaps, which have been repixelized to $0.1^{\prime}$ per pixel, normalized to the average annulus value, for each luminosity bin, for the DR5 f150 and DR5 f090 coadded maps as well as the DR4 ILC map shown for illustrative purposes only. The native units of the DR5 f150 and DR5 f090 maps are in $\mu \mathrm{K}$ and the DR4 ILC maps in $y$. Negative $y$ is plotted here to compare with the decrements present in temperature. The aperture photometry disk radius is plotted as a vertical black dashed line, and the annulus outer radius is plotted as a vertical solid black line. The beam radius is plotted as a blue vertical line for DR5 f150, an orange vertical line for DR5 f090, and a black vertical line for the effective DR4 ILC beam. A central bright spot is observed in nearly all but the most luminous bin, and is attributed to dust emission. Due to this effect, we studied the core-excised AP approach for the DR5 f150 and DR5 f090 analysis (Secs. III B and III C). 


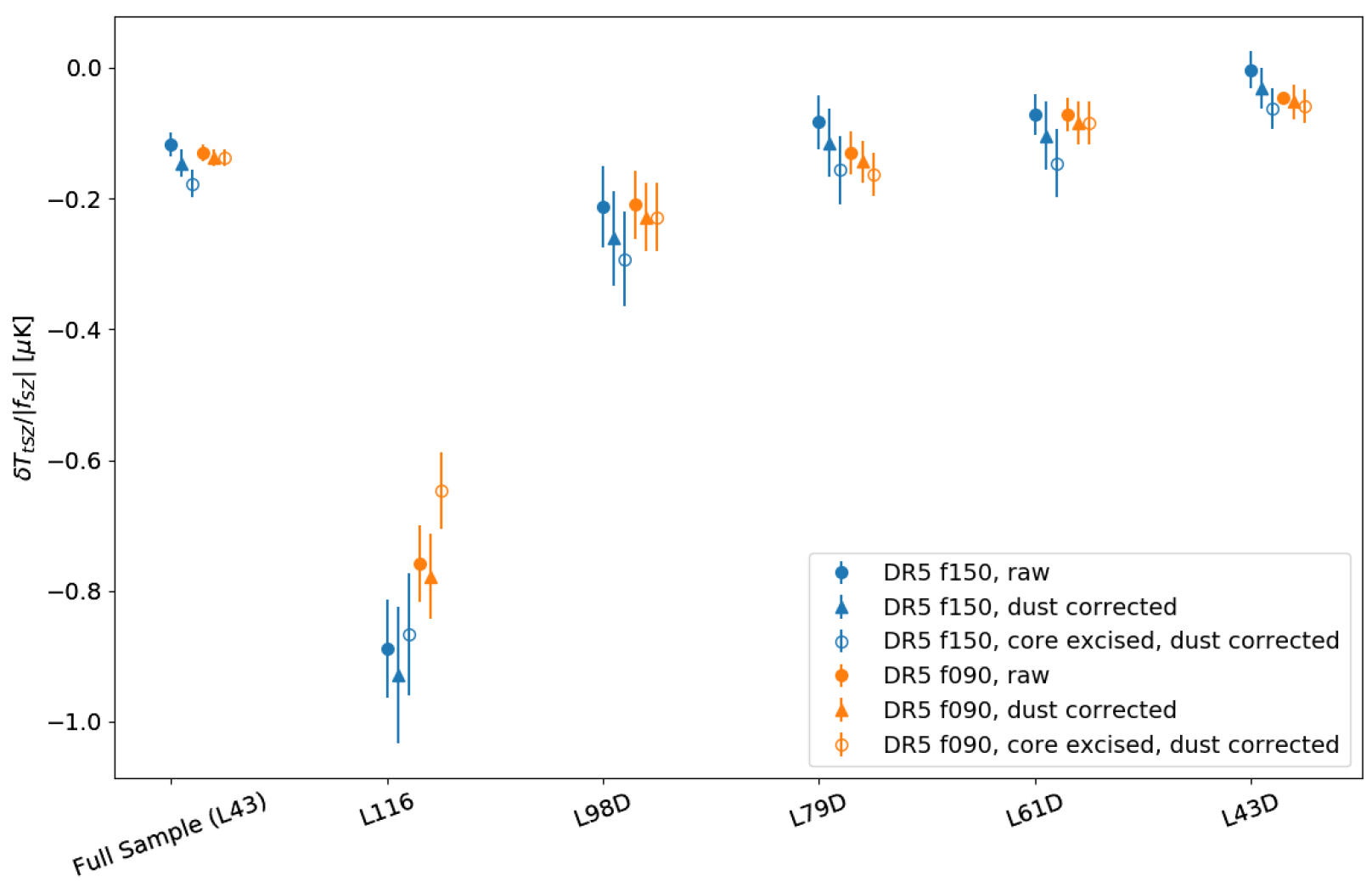

FIG. 5. Aperture photometry tSZ signals in units of temperature rescaled by $f_{\mathrm{SZ}}$ [Eq. (2)] for disjoint luminosity bins and the full analysis sample, with $1 \sigma$ jackknife estimated uncertainties, for the DR5 f150 and DR5 f090 maps. Raw results using the $2.1^{\prime}$ radius are compared to results after Herschel dust correction (Sec. III C) and the removal of the pixels within a beam-scale radius (0.7' for the DR5 f150 map and 1.1' for the DR5 f090 map; Sec. III B). The DR5 f090 results shown have been beam corrected (Sec. III B). The Herschel dust correction has a small effect on the temperature signals and lowers the signal-to-noise for the DR5 f090 map due to the relatively large error bars on the dust estimates which are propagated into the uncertainties on the tSZ signal. The "core-excised" AP approach removes a noticeable amount of tSZ signal in the L116 bin, as there is less dust contamination apparent on the beam scale in the stacked submaps for this bin as compared to the lower luminosity bins (Fig. 4). For the most part, it has less than a $1 \sigma$ effect on the tSZ signals, and is not included in the rest of this analysis. The dust-corrected (filled triangle) results are propagated through to our comparisons of optical depth estimates.

contrary to what we would expect given the larger ILC FWHM.

For the DR5 f150 and DR5 f090 analysis, we then estimate the aperture-averaged Compton- $y$ parameter $\bar{y}$ from this temperature signal and use a theoretical relation between optical depth and $\bar{y}$ from hydrodynamical simulations to infer the optical depth [3]. To obtain the Compton- $y$ parameter we follow the steps detailed in [19] and DB17. The tSZ temperature signal is related to the Compton- $y$ parameter by

$$
\frac{\delta T_{\mathrm{tSZ}}(\theta)}{T_{\mathrm{CMB}}}=f_{\mathrm{SZ}} y(\theta),
$$

where $y(\theta)$ is the Compton parameter at a projected angle $\theta$ from the cluster center and, in the nonrelativistic limit, $f_{\mathrm{SZ}}$ depends on observed radiation frequency:

$$
f_{\mathrm{SZ}}=\left(x \frac{e^{x}+1}{e^{x}-1}-4\right) \text {. }
$$

Here, $x=h \nu / k_{B} T_{\mathrm{CMB}}$ [58]. The effective band centers vary based on sky position in the coadded maps, so the median values of the SZ-weighted band centers are chosen for our analysis. The appropriately weighted band center frequencies are 97.8 and $149.6 \mathrm{GHz}$ for the DR5 f090 and DR5 f150 maps, respectively, so $f_{\mathrm{SZ}, \mathrm{f} 090}=-1.53$ and $f_{\mathrm{SZ}, \mathrm{f} 150}=$ -0.958 [59]. Relativistic corrections are negligible for this sample mass and can safely be excluded [60]. We assume the ACT-based band center frequencies because the ACT measurements dominate over those from Planck at our scales [28]. These frequencies are associated with a $2.4 \mathrm{GHz}$ uncertainty, and detailed bandpass considerations could carry a larger impact (at the few percent level). However, each of these has a small effect on $\bar{y}$ compared to our uncertainties. For each luminosity bin, $\bar{y}$ is obtained from the AP temperature signal $\delta T_{\text {tSZ }}$ via Eq. (1).

A jackknife approach is used to estimate the uncertainties associated with the binned tSZ signals and the covariance matrix for the $\mathrm{kSZ}$ analysis. We split the sample 
of sources into $N$ smaller subsamples and remove one subsample at a time, calculating the weighted average for each bin on the summed $N-1$ subsamples to obtain $N$ realizations of the computation. The jackknife method has the advantage of being self-contained and not requiring external information such as simulated CMB maps. We have varied $N$ to check the convergence of the jackknife algorithm, selecting the value that provided a stable significance against variations of $N$. For the tSZ analysis, a choice of $N=2,000$ was conservative.

In simulations with AGN feedback, Battaglia [3] finds the relationship between $\bar{y}$ and optical depth to be

$$
\ln (\bar{\tau})=\ln \left(\tau_{0}\right)+m \ln \left(\bar{y} / 10^{-5}\right)
$$

where $\ln \left(\tau_{0}\right)=-6.40$ and $m=0.49$ at $z=0.5$. We use this to estimate $\bar{\tau}$ from our $\bar{y}$ measurements. The systematic error bars on $\bar{\tau}$ from the $\bar{y}-\bar{\tau}$ relationship are calculated by using the Monte Carlo method taking into account the estimated $4 \%$ systematic uncertainty on $\ln \left(\tau_{0}\right)$ and $8 \%$ on $m$. The systematic uncertainties on $\ln \left(\tau_{0}\right)$ and $m$ were estimated in [3] by taking the largest relative differences between the radiative cooling and AGN feedback models used for Eq. (3).

A null test was performed by stacking the ILC sample on a simulated ILC CMB-only map. This test served as a check of our pipeline and jackknife estimates. The same pipeline used in the ILC analysis was used to measure the Compton-y parameter in the simulated CMB map for all luminosity bins in Table I, print stacked submaps as in Fig. 3, and plot radial averages as in Fig. 4. The Compton-y results for the simulated $\mathrm{CMB}$ map were consistent with zero within $1 \sigma$ for all luminosity bins. The stacked submaps were also consistent with the same noise level as estimated using the jackknife uncertainty estimations.

\section{C. tSZ systematic effects}

Several systematic effects have the potential to impact the amplitude of the measured tSZ signals. One potential systematic for the tSZ measurement is the light emitted from star-forming SDSS galaxies in the optical/UV that is absorbed by dust grains and reemitted in the infrared/sub$\mathrm{mm}$. To account for this, for both coadded maps we fit a model of the spectral energy distribution (SED) to Herschel data from one large extragalactic survey that overlaps with SDSS, the Herschel Astrophysical Terahertz Large Area Survey [61], in the three fields GAMA-9, GAMA-12, and GAMA-15. We use the three SPIRE photometric bands centered at 250,350 , and $500 \mu \mathrm{m}$. Table II reports the number of galaxies in the SDSS sample overlapping with the Herschel map areas for each luminosity bin analyzed.

We apply an aperture photometry filter at the position of each galaxy in our SDSS sample with aperture 2.1' and stack the signal measured from the Herschel maps following the approach described in A20 [38] (see their
TABLE II. Estimated dust signal and $1 \sigma$ statistical uncertainties for the DR5 f150 and DR5 f090 maps using 4\% of the SDSS sample used in this analysis overlapping with the Herschel map areas, as computed using the method described in [38].

\begin{tabular}{lrcc}
\hline \hline Bin & \multicolumn{1}{c}{$\mathrm{N}$} & $T_{\text {dust, } 150 \mathrm{GHz}}(\mu K)$ & $T_{\text {dust }, 98 \mathrm{GHz}}(\mu K)$ \\
\hline L43 & 12,726 & $0.026_{-0.008}^{+0.014}$ & $0.012_{-0.004}^{+0.008}$ \\
L61 & 7,784 & $0.028_{-0.010}^{+0.019}$ & $0.013_{-0.005}^{+0.010}$ \\
L79 & 3,858 & $0.029_{-0.010}^{+0.016}$ & $0.014_{-0.005}^{+0.009}$ \\
L98 & 1,795 & $0.037_{-0.014}^{+0.027}$ & $0.017_{-0.014}^{+0.014}$ \\
L116 & 941 & $0.043_{-0.003}^{+0.063}$ & $0.021_{-0.011}^{+0.031}$ \\
L43D & 4,872 & $0.026_{-0.011}^{+0.022}$ & $0.013_{-0.005}^{+0.011}$ \\
L61D & 3,926 & $0.034_{-0.017}^{+0.037}$ & $0.018_{-0.010}^{+0.023}$ \\
L79D & 2,063 & $0.027_{-0.012}^{+0.017}$ & $0.014_{-0.007}^{+0.015}$ \\
L98D & 854 & $0.049_{-0.021}^{+0.035}$ & $0.022_{-0.010}^{+0.016}$ \\
\hline \hline
\end{tabular}

Appendix B). ${ }^{1}$ Using these measurements, we fit a model of the dust SED described by the following modified blackbody:

$$
I(\nu)=A_{\mathrm{d}}\left(\frac{\nu(1+z)}{\nu_{0}}\right)^{\beta_{\mathrm{d}}+3} \frac{e^{\left(h \nu_{0} / k_{B} T_{\mathrm{d}}\right)}-1}{e^{\left(h \nu(1+z) / k_{B} T_{\mathrm{d}}\right)}-1},
$$

where $\nu_{0}=857 \mathrm{GHz}$ is the rest-frame frequency at which we normalize the dust emission, $A_{\mathrm{d}}$ is the amplitude of the dust emission in $(\mathrm{kJy} / \mathrm{sr}), \beta_{\mathrm{d}}$ is the dust spectral index, and $T_{\mathrm{d}}$ is the dust temperature in $\mathrm{K}$. We assume flat priors for the dust amplitude and temperature parameters in the ranges $0.05<A_{\mathrm{d}}(\mathrm{kJy} / \mathrm{sr})<5,10<T_{\mathrm{d}}(\mathrm{K})<40$. Given the degeneracy among the parameters, we assume a Gaussian prior for the emissivity index centered on 1.2 and with standard deviation of 0.1 , truncated in the range $1<\beta_{\mathrm{d}}<2.5$, as in [38]. We obtain constraints on the model parameters using a Markov chain Monte Carlo sampler (EMCEE [62]) to estimate the posterior probability function. Our best-fit values for the dust temperature are in the range $21<T_{\mathrm{d}}(\mathrm{K})<30$ across the luminosity bins, with $1 \sigma$ uncertainties of $\sim 30 \%$, and $\beta_{\mathrm{d}}=1.2 \pm 0.1$ consistent with our prior. We finally infer the amount of dust emission at 150 and $98 \mathrm{GHz}$. We report our estimates of the dust emission in $\mu \mathrm{K}$ units; these need to be removed from the tSZ signal in Table II. We obtain these values by multiplying our best-fit estimates from Eq. (4), with $\nu=150 \mathrm{GHz}$ and $\nu=98 \mathrm{GHz}$, respectively, by the factor $\left(\frac{d B(\nu, T)}{d T}\right)^{-1}$, where $T=T_{\mathrm{CMB}}$ and $B$ is the Planck function, in order to convert from the Herschel map intensity units to differential CMB temperature units that match the units of our SZ measurements. Because the error bars on the dust estimates are asymmetric, the largest values for each data point are selected for the more conservative estimate of $\bar{y} / \sigma(\bar{y})$ (Fig. 6).

\footnotetext{
${ }^{1}$ While A20 measure the dust profile within apertures of increasing radii, here we measure the dust signal in a single aperture of radius $2.1^{\prime}$.
} 

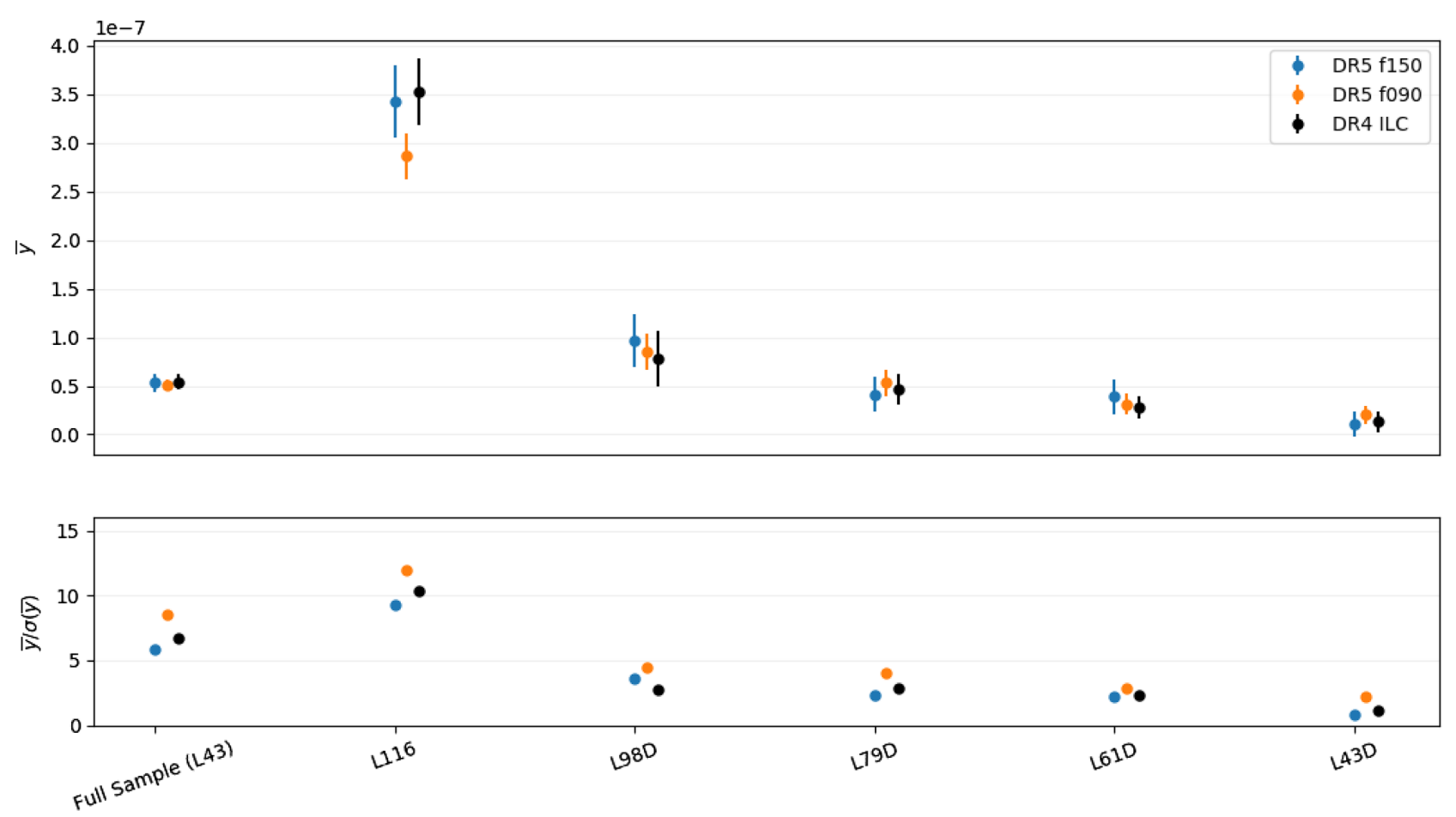

FIG. 6. Average Compton- $y$ in the 2.1' aperture for the five disjoint (highest luminosity bin L116 and bin labels with suffix "D") luminosity bins and the full source sample $\left(L>4.3 \times 10^{10} L_{\odot}\right)$, with jackknife estimated uncertainties, for the DR5 f150 and DR5 f090 maps after dust and beam correction (Secs. III B, III C, and Table II) and the DR4 ILC Compton- $y$ map after beam correction. The lower panel shows the signals divided by their associated uncertainties. The significance of the tSZ effect observed generally decreases for less luminous sources, as expected. The results from each of the three maps are consistent.

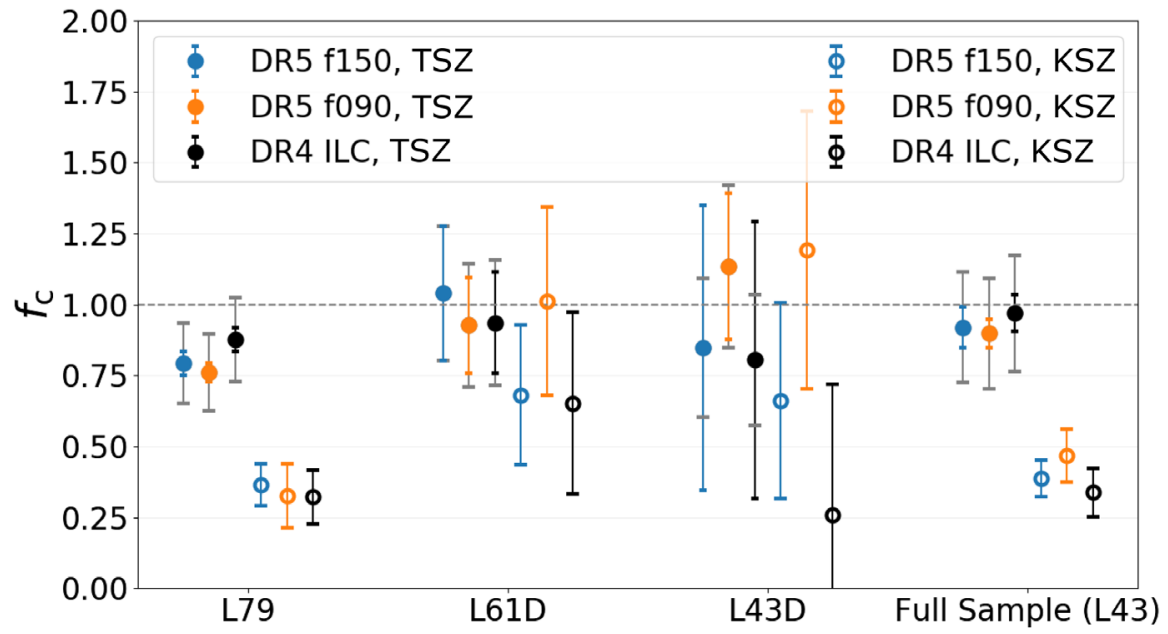

FIG. 7. Fraction of the theoretically predicted optical depth $\left(f_{\mathrm{c}}\right)$ for the full DR 15 sample. The estimates are extracted from tSZ measurements (filled circles) from three different maps (DR5 f150, blue; DR5 f090, orange; ILC, black) and kSZ measurements from the same maps, as described in C21 (open circles). The tSZ measurements are converted to optical depth estimates using a scaling relationship from hydrodynamic simulations [3]. The tSZ jackknife uncertainties are plotted in color, and the systematic uncertainties from the simulation-based scaling relationship are plotted as gray bars. The plotted kSZ uncertainties are from bootstrap estimates. The $\mathrm{kSZ}$ and tSZ results agree within $1 \sigma$ in the L61D and L43D bins, while in the highest signal-to-noise L79 bin they differ at $2-3 \sigma$. This results in the $2-3 \sigma$ difference observed in the full sample, L43. The difference between the kSZ and tSZ results is discussed in Sec. IV C. The kSZ results are lowest for the L79 bin. 


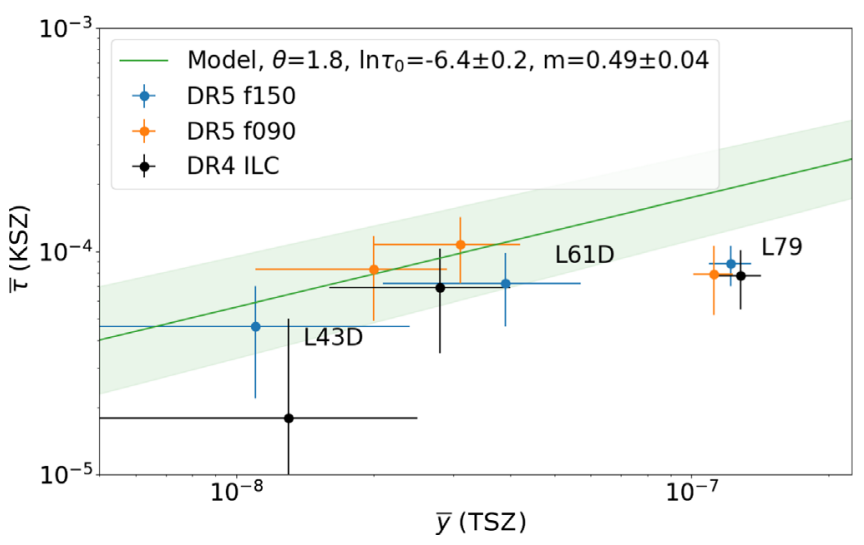

FIG. 8. Optical depth fits from kSZ signals versus average Compton- $y$ from the tSZ measurements for the three jointly analyzed disjoint luminosity bins with statistical error bars from jackknife estimates (bootstrap estimated uncertainties for the $\mathrm{kSZ}$; see $\mathrm{C} 21$ ). The scaling relation between $\bar{\tau}$ and $\bar{y}$ from hydrodynamical simulations with aperture $\Theta=1.8^{\prime}$ (the closest scaling relation in [3] to our $2.1^{\prime}$ aperture) is plotted as the green model curve [Eq. (3)] with the $1 \sigma$ uncertainty envelope shaded. The tSZ and kSZ results in the L43D and L61D luminosity bins are consistent with the model, while the kSZ results in the $\mathrm{L} 79$ bin fall below the model line.

In the raw data, we observe a central "bright spot" in several luminosity bins in all maps, most notably the DR5 f150 map. We attribute this to additional dust contamination at approximately the beam scale in the stacked submaps (Fig. 3), as shown in the radial averages presented in Fig. 4. This apparent dust contamination is particularly present in the DR5 f150 map and is stronger than the emission estimated from Herschel. The apparent angular scale comparable to the beam size suggests a compact source. We explored the effect of this excess emission by using a core-excised AP approach for the DR5 maps, using an aperture photometry filter which excluded the central disk on the beam scale (Sec. III B). We do not include the core-excised AP approach in our reported results or optical depth comparisons due to the small (generally $<1 \sigma$ ) impact on the tSZ signals, to avoid biasing our results by removing the central portion of the tSZ signal, and to best compare with the results from C21. Thus, while the Herschel dust correction accounts for some of the dust present in the DR5 f150 and DR5 f090 maps, this correction is imperfect, and some contamination remains from source galaxy emission. The effects of the dust-correction steps taken on the DR5 f150 and DR5 f090 analyses can be seen in Fig. 5.

Another potential cause of increased uncertainty in the tSZ measurements is dust and synchrotron emission from our own Galaxy. To account for this, we apply the same $50 \%$ Galactic plane mask as used in the production of the 2015 Planck all-sky Compton-y maps [31]. This cut eliminated 16,977 sources after all other cuts are considered, or $6 \%$ of our final sample, and was found to have a negligible impact on our signal compared to not applying the mask. We do not expect that synchrotron emission from the SDSS galaxies themselves has a significant impact on our discussion in this work based on the core-excised comparison shown in Fig. 5. Future work could improve upon our results by modeling and removing dust and synchrotron emission from the LRGs.

Our $\bar{y}$ estimates include a contribution from nearby halos known as the two-halo term (e.g., [63]), which biases them high when assuming we have only one halo per source. Compared to the [3] simulations, our sample includes lower mass halos and our measurements include a beam, so they will have a larger two-halo contribution. We estimate this contribution using Mop-c GT (see Appendix A of [38] for details on the implementation) and we find that our $\bar{y}$ values are biased high by a factor between $2 \%$ and $10 \%$ in the luminosity bins of interest. The two-halo bias does not significantly affect the results presented in this work, but will need to be accounted for in future higher signal-tonoise analyses.

The $\bar{y}-\bar{\tau}$ relationship [Eq. (3)] also carries associated systematics. These uncertainties were estimated based on differences between radiative cooling and AGN feedback subgrid physics models in Battaglia [3]. While this does not encompass the wide variety of existing subgrid models for the ICM, the two models are very different, and contrast in their inclusion of AGN feedback [3]. In addition to the quoted systematic uncertainty between the different models, we conjectured in DB17 that there is also uncertainty in extrapolating from the larger masses in the hydrodynamical simulations to the lower mass objects in the DR11 sample used in DB17 (Sec. IV C). New hydrodynamical simulations of lower mass clusters and groups would help address this concern.

\section{D. kSZ signal extraction}

As described in $\mathrm{C} 21$, the mean pairwise momentum of groups and clusters as a function of their comoving separation distance is negative at and around separations of $25-50 \mathrm{Mpc}$, implying that they are moving toward one another on average due to gravity [64-67]. While the threedimensional momentum of the groups is not easily measurable, the mean pairwise momentum $p$ can be still estimated from the line-of-sight component of the momenta [34]. The kSZ signal of a given cluster is directly proportional to this line-of-sight momentum: $\delta T_{\mathrm{kSZ}, \mathrm{i}} \propto-\mathbf{p}_{i} \cdot \mathbf{r}_{i}$, where the unstated multiplicative factors depend on the properties of the cluster (density profile, including angular extent in the sky) and on the pixel scale and angular resolution of the $\mathrm{CMB}$ experiment.

The significance of the kSZ measurement is determined by a fit to the analytic prediction of linear perturbation theory for the pairwise velocity $[16,17,64-68]$ rescaled by the factor $-\bar{\tau} T_{\mathrm{CMB}} / c$, where $\bar{\tau}$, the average halo optical 
depth of the galaxy sample used for the pairwise momentum estimator, is the free parameter of the fit.

\section{E. Theoretical $\bar{\tau}$ estimate}

For comparison with our measurements, we calculate theoretical estimates for the mean optical depths $\bar{\tau}_{\text {theory }}$ for each of the luminosity bins. We follow the derivation in Battaglia [3] using a Navarro-Frenk-White profile to estimate the optical depth in a given aperture,

$$
\bar{\tau}_{\text {theory }}=\sigma_{\mathrm{T}} x_{\mathrm{e}} X_{\mathrm{H}}\left(1-f_{\star}\right) f_{\mathrm{b}} \frac{M_{\mathrm{vir}}\left(<\theta_{2.1^{\prime}}\right)}{d_{A}^{2} m_{p}} .
$$

Here $\sigma_{\mathrm{T}}$ is the Thomson cross section, $x_{\mathrm{e}}$ is the electron fraction defined as $x_{\mathrm{e}}=\left(X_{\mathrm{H}}+1\right) /\left(2 X_{\mathrm{H}}\right), X_{\mathrm{H}}$ is the primordial hydrogen mass fraction $\left(X_{\mathrm{H}}=0.76\right), f_{\star}$ is the stellar mass fraction of the halo, $f_{\mathrm{b}}$ is the universal baryon fraction $\left(\Omega_{\mathrm{b}} / \Omega_{\mathrm{M}}\right), m_{\mathrm{p}}$ is the proton mass, and $d_{A}$ is the angular diameter distance to the mean redshift of our sample. The parameter value for $f_{\mathrm{b}}=0.157$ is set from the cosmological parameters we choose. The value for $f_{\star}$ is inferred from the stellar mass-halo mass relation from abundance matching as described in [51]. We define the parameter $f_{\mathrm{c}}=\bar{\tau}_{\mathrm{obs}} / \bar{\tau}_{\text {theory }}$ to compare the estimated $\bar{\tau}$ values (Table IV) to the theoretically predicted values. This parameter represents the fraction of theoretically predicted optical depth obtained by the two SZ measurements, and is of interest to compare the consistency of the two optical depth estimate methods.

\section{RESULTS}

\section{A. tSZ measurements}

Table III presents values of $\delta T_{\text {tSZ }}$ for each luminosity bin along with the averaged Compton- $\bar{y}$ parameter we calculated using the tSZ signal. These results are beam and dust corrected using our Herschel-based estimates, and assume one source per filter (Sec. III C). The signal-tonoise ratios for the tSZ measurements are up to 10 for the DR5 f150 map, 12 for the DR5 f090 map, and 10 for the ILC map, with the highest signal-to-noise in the L98 bin for the coadded maps, and the L116 bin for the ILC map. Before the Herschel dust corrections were applied and the uncertainties from those corrections propagated into the statistical tSZ uncertainty estimates, the highest signal-tonoise was seen in the highest luminosity bin (L116) for the coadded maps as well, which is as expected. The average Compton- $\bar{y}$ from the tSZ signals in the three maps is presented in Fig. 6 along with signal-to-noise per disjoint bin. The $\bar{y}$ measurements are consistent across the maps we analyzed.

The optical depths calculated using Eq. (3) range from $0.59 \pm 0.35$ (stat.) \pm 0.17 (sys.) $\times 10^{-4}$ to $3.22 \pm 0.15$ (stat.) \pm 0.42 (sys.) $\times 10^{-4}$ for all nine luminosity bins and three maps, as reported in Table IV. Potential tSZ systematic effects are discussed in Sec. III C.

\section{B. kSZ measurements}

C21 presented a value of $\bar{\tau}=(0.69 \pm 0.11) \times 10^{-4}$ as the highest signal-to-noise best-fit average optical depth. It was derived from the mean pairwise momentum for the full sample of 343,647 sources and DR5 f150 map using bootstrap uncertainty estimates of the pairwise correlation covariance matrix. In $\mathrm{C} 21$, we find that the analytical signal model is a good fit to the data with a best fit $\chi^{2}$ of 10 for 17 degrees of freedom. Selected best-fit optical depths from the mean pairwise momentum fits and bootstrap estimated signal-to-noise are reported in Table IV, and shown in Figs. 7 and 8, along with the results derived here.

TABLE III. Thermal SZ results from the DR5 f150, DR5 f090, and DR4 ILC map analyses, along with $1 \sigma$ jackknife uncertainty estimates. Dust-corrected stacked tSZ signals $\delta T_{\mathrm{tSZ}}$ and $\bar{y}$ are given for the two coadded temperature maps, and $\bar{y}$ for the DR4 ILC Compton- $y$ map. For the DR5 f150 map, the Herschel dust correction is applied, and the uncertainties associated with these corrections are propagated into the cited jackknife uncertainties. For the DR5 f090 map, the Herschel dust correction and the f090 beam correction scaling factor are applied. The $\bar{y}$ results from the disjoint bins shared with $\mathrm{C} 21$ (marked as ${ }^{+}$bins) are shown in Fig. 8.

\begin{tabular}{llllllr}
\hline \hline & \multicolumn{2}{c}{ DR5 f150 } & & \multicolumn{2}{c}{ DR5 f090 } & DR4 ILC \\
\cline { 2 - 3 } Bin & $\delta \mathrm{T}_{\text {tSZ,corr. }}(\mu \mathrm{K})$ & $\bar{y} / 10^{-7}$ & & $\delta \mathrm{T}_{\text {tSZ,corr. }}(\mu \mathrm{K})$ & $\bar{y} / 10^{-7}$ & $\bar{y} / 10^{-7}$ \\
\hline L43 & $-0.14 \pm 0.02$ & $0.53 \pm 0.09$ & & $-0.21 \pm 0.02$ & $0.51 \pm 0.06$ & $0.54 \pm 0.08$ \\
L61 & $-0.21 \pm 0.03$ & $0.79 \pm 0.11$ & & $-0.29 \pm 0.03$ & $0.70 \pm 0.07$ & $0.78 \pm 0.10$ \\
L79 $^{+}$ & $-0.32 \pm 0.03$ & $1.22 \pm 0.13$ & & $-0.47 \pm 0.04$ & $1.12 \pm 0.11$ & $1.28 \pm 0.14$ \\
L98 $_{\text {L116 }}$ & $-0.57 \pm 0.06$ & $2.18 \pm 0.22$ & & $-0.77 \pm 0.06$ & $1.84 \pm 0.15$ & $2.19 \pm 0.22$ \\
L43D $^{+}$ & $-0.89 \pm 0.10$ & $3.42 \pm 0.37$ & & $-1.19 \pm 0.10$ & $2.86 \pm 0.24$ & $3.52 \pm 0.34$ \\
L61D $^{+}$ & $-0.03 \pm 0.03$ & $0.11 \pm 0.13$ & & $-0.08 \pm 0.04$ & $0.20 \pm 0.09$ & $0.13 \pm 0.11$ \\
L79D & $-0.10 \pm 0.05$ & $0.39 \pm 0.18$ & & $-0.13 \pm 0.05$ & $0.31 \pm 0.11$ & $0.28 \pm 0.12$ \\
L98D & $-0.11 \pm 0.05$ & $0.41 \pm 0.18$ & & $-0.22 \pm 0.05$ & $0.53 \pm 0.13$ & $0.46 \pm 0.16$ \\
\hline \hline
\end{tabular}


TABLE IV. Optical depth estimates from the tSZ effect via hydrodynamic simulations, $1 \sigma$ statistical and systematic uncertainties, and fraction of theoretical estimates for mean optical depths ( $f_{c}$, Sec. III E) for each luminosity bin and analyzed map. Statistical uncertainties on tSZ estimated optical depth are propagated from the tSZ AP jackknife uncertainty estimates and Hershcel dust corrections. Systematic uncertainties are estimated using the Monte Carlo method taking into account the estimated systematic uncertainties in the $\bar{y}-\bar{\tau}$ relationship from simulations [Eq. (3)]. For example, the $\bar{\tau}$ fit from the DR5 f150 tSZ results for $L>4.30 \times 10^{10} L_{\odot}, 1.28 \times 10^{-4}$, divided by the theoretical $\bar{\tau}$ estimate of $1.39 \times 10^{-4}$, yields $f_{\mathrm{c}}=0.92$ for that galaxy sample. Selected $\bar{\tau}$ estimates from the pairwise kSZ effect from C21 with bootstrap uncertainties are listed along with $f_{\mathrm{c}}$ for comparison. The fractions for the full galaxy sample ( ${ }^{* *}$ bin) are shown in Fig. 7, and kSZ results from the three disjoint bins shared with $\mathrm{C} 21\left({ }^{+}\right.$bins) are shown in Fig. 8. Uncertainties on mass estimates from luminosity have not been propagated through to the $\bar{\tau}_{\text {theory }}$ estimate, so $f_{\mathrm{c}}$ is best used for comparison and the study of relative trends.

\begin{tabular}{|c|c|c|c|c|c|c|}
\hline & \multirow[b]{2}{*}{$\bar{\tau}_{\text {theory }}$} & \multicolumn{5}{|c|}{ DR5 f150 } \\
\hline & & $\bar{\tau}_{\mathrm{tSZ}}$ & $\sigma_{\text {sys }}$ & & $\bar{\tau}_{\mathrm{kSZ}}(\mathrm{C} 21)$ & \\
\hline Bin & $\left(10^{-4}\right)$ & $\left(10^{-4}\right)$ & $\left(10^{-4}\right)$ & $f_{c, \mathrm{tSZ}} \pm$ (stat., sys.) & $\left(10^{-4}\right)$ & $f_{c, \mathrm{kSZ}}$ \\
\hline $\mathrm{L} 3^{* *}$ & 1.39 & $1.28 \pm 0.10$ & 0.27 & $0.92 \pm(0.07,0.20)$ & $0.54 \pm 0.09$ & $0.39 \pm 0.06$ \\
\hline L61 & 1.77 & $1.55 \pm 0.11$ & 0.30 & $0.88 \pm(0.06,0.17)$ & $0.69 \pm 0.11$ & $0.39 \pm 0.06$ \\
\hline $\mathrm{L} 79^{+}$ & 2.42 & $1.92 \pm 0.10$ & 0.34 & $0.79 \pm(0.04,0.14)$ & $0.88 \pm 0.18$ & $0.36 \pm 0.07$ \\
\hline L98 & 3.35 & $2.55 \pm 0.12$ & 0.39 & $0.76 \pm(0.04,0.12)$ & & \\
\hline L116 & 4.44 & $3.18 \pm 0.17$ & 0.43 & $0.72 \pm(0.04,0.10)$ & & \\
\hline $\mathrm{L}_{43 \mathrm{D}^{+}}$ & 0.70 & $0.59 \pm 0.35$ & 0.17 & $0.85 \pm(0.50,0.24)$ & $0.46 \pm 0.24$ & $0.66 \pm 0.34$ \\
\hline $\mathrm{L} 61 D^{+}$ & 1.06 & $1.10 \pm 0.25$ & 0.25 & $1.04 \pm(0.24,0.23)$ & $0.72 \pm 0.26$ & $0.68 \pm 0.25$ \\
\hline L79D & 1.53 & $1.12 \pm 0.24$ & 0.25 & $0.74 \pm(0.16,0.16)$ & & \\
\hline L98D & 2.09 & $1.71 \pm 0.23$ & 0.33 & $0.82 \pm(0.11,0.16)$ & & \\
\hline
\end{tabular}

\begin{tabular}{|c|c|c|c|c|c|c|}
\hline & \multirow[b]{2}{*}{$\bar{\tau}_{\text {theory }}$} & \multicolumn{5}{|c|}{ DR5 f090 } \\
\hline & & $\bar{\tau}_{\mathrm{tSZ}}$ & $\sigma_{\text {sys }}$ & & $\bar{\tau}_{\mathrm{kSZ}}(\mathrm{C} 21)$ & \\
\hline Bin & $\left(10^{-4}\right)$ & $\left(10^{-4}\right)$ & $\left(10^{-4}\right)$ & $f_{c, \mathrm{tSZ}} \pm$ (stat., sys.) & $\left(10^{-4}\right)$ & $f_{c, \mathrm{kSZ}}$ \\
\hline $\mathrm{L} 43^{* *}$ & 1.39 & $1.25 \pm 0.07$ & 0.27 & $0.89 \pm(0.05,0.19)$ & $0.65 \pm 0.13$ & $0.47 \pm 0.09$ \\
\hline L61 & 1.77 & $1.46 \pm 0.07$ & 0.29 & $0.82 \pm(0.04,0.16)$ & $0.82 \pm 0.17$ & $0.46 \pm 0.10$ \\
\hline${\mathrm{L} 79^{+}}^{+}$ & 2.42 & $1.84 \pm 0.08$ & 0.32 & $0.76 \pm(0.03,0.13)$ & $0.79 \pm 0.27$ & $0.33 \pm 0.11$ \\
\hline L98 & 3.35 & $2.35 \pm 0.10$ & 0.37 & $0.70 \pm(0.03,0.11)$ & & \\
\hline L116 & 4.44 & $2.91 \pm 0.12$ & 0.41 & $0.66 \pm(0.03,0.09)$ & & \\
\hline $\mathrm{L}_{43 \mathrm{D}^{+}}$ & 0.70 & $0.79 \pm 0.18$ & 0.20 & $1.14 \pm(0.25,0.29)$ & $0.83 \pm 0.34$ & $1.19 \pm 0.49$ \\
\hline $\mathrm{L} 61 D^{+}$ & 1.06 & $0.98 \pm 0.18$ & 0.23 & $0.92 \pm(0.17,0.22)$ & $1.07 \pm 0.35$ & $1.01 \pm 0.33$ \\
\hline L79D & 1.53 & $1.27 \pm 0.15$ & 0.27 & $0.83 \pm(0.10,0.18)$ & & \\
\hline L98D & 2.09 & $1.60 \pm 0.18$ & 0.30 & $0.77 \pm(0.09,0.15)$ & & \\
\hline
\end{tabular}

\begin{tabular}{|c|c|c|c|c|c|c|}
\hline & \multirow[b]{2}{*}{$\bar{\tau}_{\text {theory }}$} & \multicolumn{5}{|c|}{ DR4 ILC } \\
\hline & & $\bar{\tau}_{\mathrm{tSZ}}$ & $\sigma_{\text {sys }}$ & & $\bar{\tau}_{\mathrm{kSZ}}(\mathrm{C} 21)$ & \\
\hline Bin & $\left(10^{-4}\right)$ & $\left(10^{-4}\right)$ & $\left(10^{-4}\right)$ & $f_{c, \mathrm{tSZ}} \pm$ (stat., sys.) & $\left(10^{-4}\right)$ & $f_{c, \mathrm{kSZ}}$ \\
\hline $\mathrm{L}^{4} 3^{* *}$ & 1.39 & $1.29 \pm 0.09$ & 0.27 & $0.92 \pm(0.07,0.19)$ & $0.47 \pm 0.12$ & $0.34 \pm 0.09$ \\
\hline L61 & 1.77 & $1.54 \pm 0.09$ & 0.30 & $0.87 \pm(0.05,0.17)$ & $0.74 \pm 0.15$ & $0.42 \pm 0.08$ \\
\hline $\mathrm{L} 9^{+}$ & 2.42 & $1.96 \pm 0.11$ & 0.34 & $0.81 \pm(0.04,0.14)$ & $0.78 \pm 0.23$ & $0.32 \pm 0.10$ \\
\hline L98 & 3.35 & $2.55 \pm 0.13$ & 0.39 & $0.76 \pm(0.04,0.12)$ & & \\
\hline L116 & 4.44 & $3.22 \pm 0.15$ & 0.43 & $0.73 \pm(0.03,0.10)$ & & \\
\hline $\mathrm{L}_{43 \mathrm{D}^{+}}$ & 0.70 & $0.64 \pm 0.26$ & 0.17 & $0.91 \pm(0.37,0.25)$ & $0.18 \pm 0.32$ & $0.26 \pm 0.46$ \\
\hline $\mathrm{L} 61 D^{+}$ & 1.06 & $0.93 \pm 0.20$ & 0.22 & $0.88 \pm(0.19,0.21)$ & $0.69 \pm 0.34$ & $0.65 \pm 0.32$ \\
\hline L79D & 1.53 & $1.19 \pm 0.20$ & 0.26 & $0.78 \pm(0.13,0.17)$ & & \\
\hline L98D & 2.09 & $1.54 \pm 0.27$ & 0.30 & $0.74 \pm(0.13,0.14)$ & & \\
\hline
\end{tabular}

\section{Optical depth comparisons}

Optical depth estimates from the two SZ effects are presented in Table IV along with comparisons to theory. Figure 7 presents the fraction of theoretically predicted optical depth $\left(f_{\mathrm{c}}\right)$ for the three independent joint analysis bins and the full sample, a representation of the baryon fraction we observe within the AP radius for those source samples. The tSZ and kSZ estimates are consistent within 
$1 \sigma$ for the two lower mass disjoint bins (L61D and L43D), while they differ at $2-3 \sigma$ in the highest mass bin (L79), which is also the best constrained. This difference drives $2-3 \sigma$ differences between the tSZ and $\mathrm{kSZ}$ results in the cumulative bins. This difference may be decreased if the fixed cosmology assumed for the kSZ optical depth fits were allowed to vary. We look forward to comparing the two tracers in more detail in future work with even more sensitive datasets. The addition of more disjoint bins with sufficient numbers of sources for high signal-to-noise kSZ measurements will aid in the interpretation of any massdependent effects. Additionally, the halo mass input in the kSZ theory fits in C21 will continue to be investigated in future analyses. The comparisons with theoretical estimates of the optical depth suggest that between one-third and all of the predicted baryons lie within the aperture size of radius $2.1^{\prime}$ or $1.1 \mathrm{Mpc}$ (for a mean redshift of $z=0.55$ ) studied in this analysis. However, because uncertainties in the luminosity-mass relation for DR15 (Sec. III E) are difficult to accurately propagate through to $\bar{\tau}_{\text {theory }}$, it is challenging to draw strong conclusions about baryon content based on our estimates of $f_{\mathrm{c}}$. For future high signal-to-noise measurements, careful treatment of the uncertainties discussed in [51] will be necessary for accurate interpretation of these SZ results. Here, the $f_{\mathrm{c}}$ values are used for making comparisons between the SZ estimates and studying trends.

Figure 8 shows a comparison of $\bar{\tau}$ estimates from kSZ measurements and average Compton- $y$ from tSZ measurements to the power-law scaling relation using an AGN feedback model simulation relation [Eq. (3)] [3]. We expect significant covariance between the data from the different maps. Two of the three disjoint analysis bins are in agreement with the model, while the highest mass bin (L79) is an outlier. This appears to be driven by low $\mathrm{kSZ} \bar{\tau}$ estimates for this bin (Fig. 7).

The two-halo contribution to our tSZ measurements discussed in Sec. IIIC does not affect these results. Propagating our $\bar{y}$ corrections to $\bar{\tau}_{\mathrm{tSZ}}$ and then to $f_{c, \mathrm{tSZ}}$ (filled circles in Fig. 8), we get lower tSZ fractions by a factor of $4 \%$ for the L43D bin and less for the other bins.

Our fits represent a step toward empirical $\bar{y}-\bar{\tau}$ relationships from a fit to kSZ and tSZ measurements. These measurements will thus serve as tests and checks for current and future cosmological simulations, and improve our understanding of galaxy formation and feedback models.

\section{CONCLUSION}

We have presented estimates of halo optical depths from measurements of the tSZ effect made on the most recent multifrequency ACT + Planck maps in combination with LRG tracers from the SDSS BOSS DR15 catalog. By combining them with the $\mathrm{kSZ}$ measurements presented in C21, we have compared estimates of optical depths from the two SZ effects and make progress toward empirical $\bar{y}-\bar{\tau}$ relationships from the SZ effects. We have improved our approach compared to our previous work, DB17, by analyzing the CMB map with the same AP filter for both the $\mathrm{kSZ}$ and $\mathrm{tSZ}$ analysis, and removing contaminating dust emission by estimating the contribution from dusty starforming galaxies using Herschel maps and a modified blackbody dust emission model.

The stacked tSZ signals have been converted to an estimate of optical depth through a hydrodynamic simulation scaling model [3], while the pairwise kSZ signals have been fit to theoretical predictions to find best-fit optical depths (C21). The two methods are independent of one another, and each of the SZ results is consistent over the three maps analyzed (DR5 f150, DR5 f090, and DR4 ILC). The results from the tSZ and kSZ measurements agree with one another within $1 \sigma$ in the two lower mass disjoint bins, while they differ by $2-3 \sigma$ in the highest mass bin and thus the cumulative bin. Across all bins, the optical depth estimates from the SZ effects account for onethird to all of the theoretically predicted baryon content. When comparing the tSZ $\bar{y}$ measurements and $\mathrm{kSZ} \bar{\tau}$ results to the hydrodynamic model, two of the three bins analyzed are in agreement with the model.

Using tSZ data appears to be a promising approach for obtaining accurate estimates of galaxy group and cluster optical depths and eventually estimating the mean pairwise velocity from pairwise momentum measurements, once a better understanding of the relationship between $\bar{y}$ and $\bar{\tau}$ is achieved. Our results help to move us from an era of measurement alone to one of interpretation based on the consistency of previously inaccessible quantities.

With improved data from the complete ACT dataset [69] and current and upcoming projects such as CCAT-prime [70], the Simons Observatory [71], DESI [72], SPT-3G [73] and CMB-S4 [74], we will achieve higher signal-to-noise measurements of the SZ signals and be able to probe the baryon content of galaxy clusters and groups and largescale structure further. Improved multifrequency data will enable precise measurements of optical depths and peculiar velocities simultaneously for large samples and for single sources, potentially sensitive enough to measure the missing baryons between groups. With these data, the SZ signals are anticipated to become valuable cosmological probes that are complementary to current observables.

\section{ACKNOWLEDGMENTS}

E. M. V. acknowledges support from the NSF Graduate Research Fellowship Program under Grant No. DGE1650441. M.D. N. acknowledges support from NSF CAREER Grant No. 1454881. V. C. and R. B. acknowledge support from DOE Grant No. DE-SC0011838, NASA ATP Grant No. 80NSSC18K0695, NASA ROSES Grant No. 12EUCLID12-0004, and funding related to the Roman High 
Latitude Survey Science Investigation Team. N. B. acknowledges support from NSF Grant No. AST-1910021, NASA ATP Grant No. 80NSSC18K0695, and from the Research and Technology Development fund at the Jet Propulsion Laboratory through the project titled "Mapping the Baryonic Majority." E. C. acknowledges support from the STFC Ernest Rutherford Fellowship No. ST/M004856/2 and STFC Consolidated Grant No. ST/S00033X/1, and from the European Research Council under the European Union's Horizon 2020 research and innovation program (Grant Agreement No. 849169). S. K.C. acknowledges support from NSF Grant No. AST-2001866. J. D. is supported through NSF Grant No. AST-1814971. R. D. thanks CONICYT for Grant No. BASAL CATA AFB-170002. R.H. acknowledges funding from the CIFAR Azrieli Global Scholars program and the Alfred P. Sloan Foundation. J. P. H. acknowledges funding for SZ cluster studies from NSF Grant No. AST-1615657. K. M. acknowledges support from the National Research Foundation of South Africa. C. S. acknowledges support from the Agencia Nacional de Investigación y Desarrollo through FONDECYT Iniciación Grant No. 11191125. Z.X. is supported by the Gordon and Betty Moore Foundation. This work was supported by the U.S. National Science Foundation through Grants No. AST-0408698, No. AST0965625, and No. AST-1440226 for the ACT project, as well as Grants No. PHY-0355328, No. PHY-0855887, and No. PHY-1214379. Funding was also provided by Princeton University, the University of Pennsylvania, and a Canada Foundation for Innovation grant to U. B. C. ACT operates in the Parque Astronómico Atacama in northern Chile under the auspices of the Comisión Nacional de Investigación Científica y Tecnológica de Chile. Canadian co-authors acknowledge support from the Natural Sciences and Engineering Research Council of Canada. The Dunlap Institute is funded through an endowment established by the David Dunlap family and the University of Toronto. Funding for the Sloan Digital Sky Survey IV has been provided by the Alfred P. Sloan Foundation, the U.S. Department of Energy Office of Science, and the Participating Institutions. SDSSIV acknowledges support and resources from the Center for High Performance Computing at the University of Utah. SDSS-IV is managed by the Astrophysical Research Consortium for the Participating Institutions of the SDSS Collaboration including the Brazilian Participation Group, the Carnegie Institution for Science, Carnegie Mellon
University, Center for Astrophysics-Harvard and Smithsonian, the Chilean Participation Group, the French Participation Group, Instituto de Astrofísica de Canarias, The Johns Hopkins University, Kavli Institute for the Physics and Mathematics of the Universe/University of Tokyo, the Korean Participation Group, Lawrence Berkeley National Laboratory, Leibniz Institut für Astrophysik Potsdam, MaxPlanck-Institut für Astronomie Heidelberg, Max-PlanckInstitut für Astrophysik Garching, Max-Planck-Institut für Extraterrestrische Physik, National Astronomical Observatories of China, New Mexico State University, New York University, University of Notre Dame, Observatário Nacional/MCTI, The Ohio State University, Pennsylvania State University, Shanghai Astronomical Observatory, United Kingdom Participation Group, Universidad Nacional Autónoma de México, University of Arizona, University of Colorado Boulder, University of Oxford, University of Portsmouth, University of Utah, University of Virginia, University of Washington, University of Wisconsin, Vanderbilt University, and Yale University.

\section{APPENDIX A: CASJOBS SDSS QUERY}

The SDSS data used in this work and C21 were downloaded from the SDSS Catalog Archive Server via the SkyServer website in May of 2019. All of the CMASS and LOWZ galaxies should be included in this catalog, in addition to more recently released eBOSS galaxies. We use this approach in defining the catalog to utilize as many SDSS LRGs as possible. The following query returned 602,461 objects. The positions, redshifts, Cmodel and Petrosian magnitudes, extinction corrections, and object IDs are recorded for the objects. A RA (right ascension) and DEC (declination) cut is applied to target the survey area overlapping with our maps, and the redshifts of the objects are selected to match the range in DB17. "ZWARNING_NOQSO $=0$ " indicates that the automated redshift estimate is reliable for the source. Objects with "zWarning $=0 "$ have no known redshift issues. Querying with "sciencePrimary $>0$ " selects the best available unique set of spectra for the objects. Bitmasks are applied to exclude SDSS target flags: "SpecObjAll.TILE >= 10324" excludes incorrectly targeted LOWZ galaxies in early BOSS data, and the i-band fiber magnitude is selected to be the same as in DB17.

\section{SELECT}

SpecObjAll.ra, SpecObjAll.dec, SpecObjAll.z, PhotoObjAll. cModelMag_u, PhotoObjAll. cModelMag_g, PhotoObjAll. cModelMag_r, PhotoObjAll. cModelMag_i, PhotoObjAll. cModelMag_z, PhotoObjAll. cModelMagErr_u, PhotoObjAll.

cModelMagErr_g, PhotoObjAll.c ModelMagErr_r, PhotoObjAll. cModelMagErr_i, PhotoObjAll. cModelMagErr_z, PhotoObjAll. petroMag_u, PhotoObjAll. petroMag_g, PhotoObjAll. petroMag_r, PhotoObjAll. 
petroMag_i,PhotoObjAll. petroMag_z,PhotoObjAll. petroMagErr_u,PhotoObjAll.

petroMagErr_g,

PhotoObjAll. petroMagErr_r, PhotoObjAll. petroMagErr_i, PhotoObjAll. petroMagErr_z,

PhotoObjAll. extinction_u, PhotoObjAll. extinction_g, PhotoObjAll. extinction_r,

PhotoObjAll. extinction_i,

PhotoObjAll. extinction_z, SpecObjAll. bestObjID into

DR15_actplanck_catalog_wbestObjID_PetrANDcModel_20200902_EMV from SpecObjAll,

PhotoObjAll, Photoz

WHERE

$(($ SpecObjAll. bestObjID = PhotoObjAll.objID $)$ and $($ SpecObjAll. bestObjID $=$ Photoz.objID ) )

and

((((SpecObjAll. ra BETWEEN 142.0 AND 180.0) and (SpecObjAll. dec BETWEEN -8.3 AND

22.0)) or ((SpecObjAll. ra BETWEEN 0.0 AND 142.0) and (SpecObjAll. dec BETWEEN

-61.5 AND 22.0))

or ((SpecObjAll. ra BETWEEN 246.0 AND 360.0) and (SpecObjAll. dec BETWEEN -61.5

AND 22.0)) or ((SpecObjAll. ra BETWEEN 180.0 AND 246.0) and (SpecObjAll. dec

BETWEEN -8.3 AND 22.0)))

and $($ SpecObjAll. ZWARNING_NOQSO $=0)$ and

$($ SpecObjAll. zWarning $=0)$ AND

(SpecObjAll. sciencePrimary $>0$ ) and

(SpecObjAll. $\mathrm{z}>0.049$ ) and

(SpecObjAll. $\mathrm{z}<0.8)$ and

$(((($ SpecObjAll. BOSS_TARGET1 $\& 0 \times 0000000000000001) !=0)$ and

(SpecObjAll. TILE $>=10324)$ ) OR

$((($ SpecObjAll. BOSS_TARGET1 \& $0 \times 0000000000000002) !=0)$ and

$($ PhotoObjAll. fiber2Mag_i $<21.5)))$ )

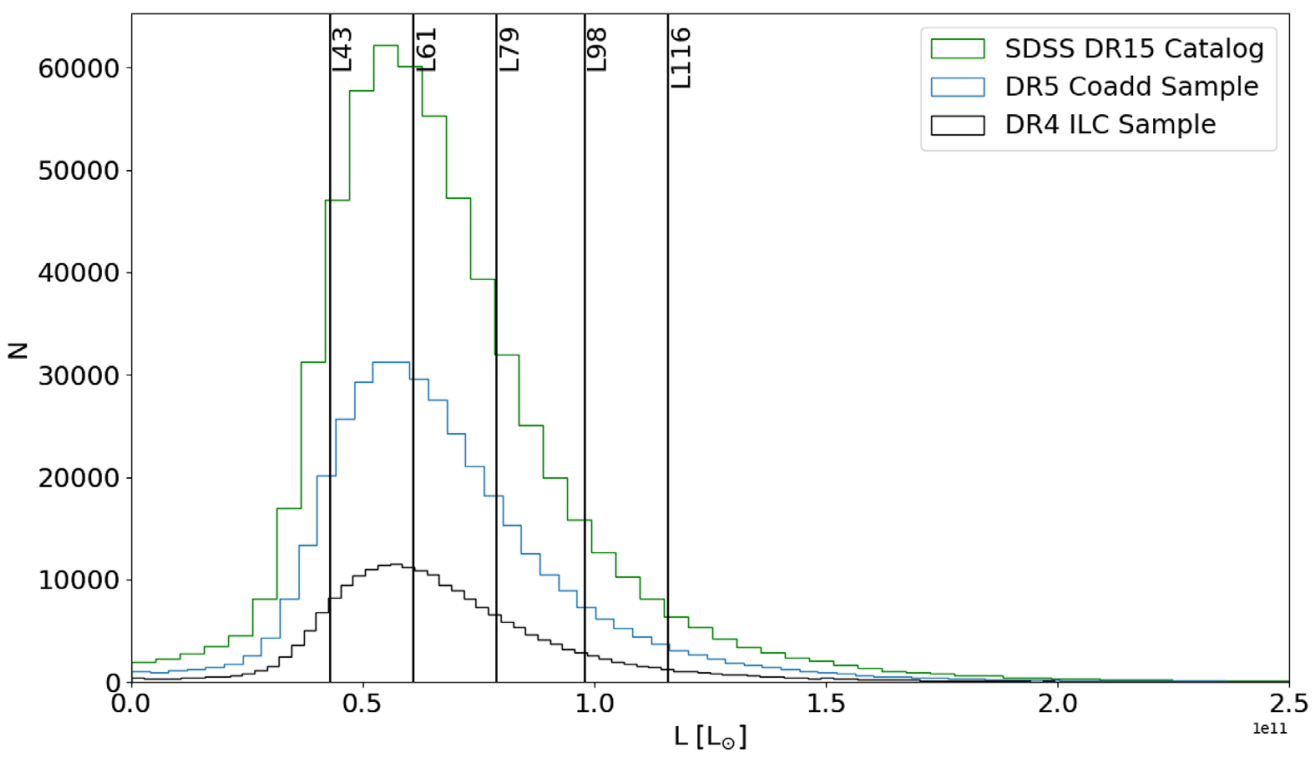

FIG. 9. Luminosity bin cuts for the SDSS DR15 catalog plotted over a histogram of the full sample (green), DR5 f150 and DR5 f090 selected analysis sample (blue), and DR4 ILC sample (black). The bottom three bins were selected to each have over 100,000 galaxies for the joint tSZ and kSZ analyses of the DR5 maps, while being roughly evenly spaced and overlapping with bin selection from the DB17 analysis. The top two bins were added for the tSZ analysis to study higher mass bins that have a strong tSZ signal, while also overlapping with DB17 bins. 


\section{APPENDIX B: LUMINOSITY BINNING}

As discussed in Sec. II B, luminosity bins for the joint tSZ and kSZ analyses with $\mathrm{C} 21$ were chosen based on luminosity cuts from DB17 $\left(L>7.9 \times 10^{10} L_{\odot}\right.$ and $\left.L>6.1 \times 10^{10} L_{\odot}\right)$ as well as one lower luminosity cut,
$\left(L>4.3 \times 10^{10} L_{\odot}\right)$. The three disjoint bins were selected to have roughly equal spacing, each with $\sim 100,000$ galaxies after cutting for analysis with the DR5 maps (Table I). The luminosity cuts are plotted over a histogram of the SDSS samples in Fig. 9.
[1] Y. B. Zeldovich and R. A. Sunyaev, Astrophys. Space Sci. 4, 301 (1969).

[2] R. A. Sunyaev and Y. B. Zeldovich, Comments Astrophys. Space Phys. 4, 173 (1972).

[3] N. Battaglia, J. Cosmol. Astropart. Phys. 8 (2016) 058.

[4] T. Mroczkowski et al., Bull. Am. Astron. Soc. 51, 124 (2019).

[5] M. Fukugita and P. J. E. Peebles, Astrophys. J. 616, 643 (2004).

[6] F. Nicastro, S. Mathur, and M. Elvis, Science 319, 55 (2008).

[7] J. M. Shull, B. D. Smith, and C. W. Danforth, Astrophys. J. 759, 23 (2012).

[8] B. Rasheed, N. Bahcall, and P. Bode, Proc. Natl. Acad. Sci. U.S.A. 108, 3487 (2011).

[9] I. Chiu, J. J. Mohr, M. McDonald, S. Bocquet et al., Mon. Not. R. Astron. Soc. 478, 3072 (2018).

[10] E. Schaan et al., Phys. Rev. D 93, 082002 (2016).

[11] S. Ho, S. DeDeo, and D. Spergel, arXiv:0903.2845.

[12] P. Richter, B. D. Savage, T. M. Tripp, and K. R. Sembach, arXiv:astro-ph/0412133.

[13] R. Cen and J. P. Ostriker, Astrophys. J. 650, 560 (2006).

[14] J. Chaves-Montero, C. Hernandez-Monteagudo, R. E. Angulo, and J. D. Emberson, Mon. Not. R. Astron. Soc. 503, 1798 (2021).

[15] N. Hand et al. (ACT Collaboration), Phys. Rev. Lett. 109, 041101 (2012).

[16] E.-M. Mueller, F. de Bernardis, R. Bean, and M. D. Niemack, Phys. Rev. D 92, 063501 (2015).

[17] E.-M. Mueller, F. de Bernardis, R. Bean, and M. D. Niemack, Astrophys. J. 808, 47 (2015).

[18] T. A. Marriage et al. (ACT Collaboration), Astrophys. J. 737, 61 (2011).

[19] M. Hasselfield et al. (ACT Collaboration), J. Cosmol. Astropart. Phys. 07 (2013) 008.

[20] M. Hilton, M. Hasselfield, C. Sifn, N. Battaglia et al. (ACT Collaboration), Astrophys. J. Suppl. Ser. 235, 20 (2018).

[21] N. Aghanim, M. Douspis, G. Hurier, D. Crichton et al., Astron. Astrophys. 632, A47 (2019).

[22] Z. Staniszewski et al., Astrophys. J. 701, 32 (2009).

[23] L. Bleem, B. Stalder, T. de Haan et al., Astrophys. J. Suppl. Ser. 216, 27 (2015).

[24] Planck Collaboration, Astron. Astrophys. 641, A1 (2020).

[25] B. J. Fuzia et al. (ACT Collaboration), Mon. Not. R. Astron. Soc. 502, 4026 (2021).

[26] M. B. Gralla et al., Mon. Not. R. Astron. Soc. 445, 460 (2014).

[27] T. Su et al., Mon. Not. R. Astron. Soc. 464, 968 (2017).
[28] S. Naess et al. (ACT Collaboration), J. Cosmol. Astropart. Phys. 12 (2020) 046.

[29] M. S. Madhavacheril, J. C. Hill, S. Næss et al. (ACT Collaboration), Phys. Rev. D 102, 023534 (2020).

[30] D. S. Aguado et al., Astrophys. J. Suppl. Ser. 240, 23 (2019).

[31] Planck Collaboration, Astron. Astrophys. 594, A22 (2016).

[32] V. Calafut, P. A. Gallardo, E. M. Vavagiakis, S. Amodeo et al. (ACT Collaboration), preceding paper, Phys. Rev. D 104, 043502 (2021)..

[33] F. De Bernardis et al. (ACT Collaboration), J. Cosmol. Astropart. Phys. 03 (2017) 008.

[34] P. G. Ferreira, R. Juszkiewicz, H. A. Feldman, M. Davis, and A. H. Jaffe, Astrophys. J. 515, L1 (1999).

[35] Planck Collaboration, Astron. Astrophys. 586, A140 (2016).

[36] B. Soergel et al., Mon. Not. R. Astron. Soc. 461, 3172 (2016).

[37] N. Hand et al., Astrophys. J. 736, 39 (2011).

[38] S. Amodeo et al. (ACT Collaboration), Phys. Rev. D 103, 063514 (2021).

[39] E. Schaan et al. (ACT Collaboration), Phys. Rev. D 103, 063513 (2021).

[40] C. P. Ahn et al., Astrophys. J. Suppl. Ser. 211, 17 (2014).

[41] R. J. Thornton et al. (ACT Collaboration), Astrophys. J. Suppl. Ser. 227, 21 (2016).

[42] https://www.sdss.org/dr15/.

[43] K. S. Dawson et al., Astron. J. 145, 10 (2013).

[44] V. Calafut, R. Bean, and B. Yu, Phys. Rev. D 96, 123529 (2017).

[45] https://github.com/evavagiakis/V21_Catalog/.

[46] http://kcorrect.org.

[47] M. R. Blanton and S. Roweis, Astron. J. 133, 734 (2007).

[48] R. H. Lupton, J. E. Gunn, and A. S. Szalay, Astron. J. 118, 1406 (1999).

[49] S. K. Choi et al. (ACT Collaboration), J. Cosmol. Astropart. Phys. 12 (2020) 045.

[50] G. Chabrier, Publ. Astron. Soc. Pac. 115, 763 (2003).

[51] A. Kravtsov, A. Vikhlinin, and A. Meshcheryakov, Astron. Lett. 44, 8 (2018).

[52] E. F. Bell and R. S. de Jong, Astrophys. J. 550, 212 (2001).

[53] E. F. Bell, D. H. McIntosh, N. Katz, and M. D. Weinberg, Astrophys. J. Suppl. Ser. 149, 289 (2003).

[54] M. Bernardi, F. Shankar, J. B. Hyde, S. Mei, F. Marulli, and R. K. Sheth, Mon. Not. R. Astron. Soc. 404, 2087 (2010).

[55] https://github.com/simonsobs/pixell.

[56] N. Battaglia, J. R. Bond, C. Pfrommer, and J. L. Sievers, Astrophys. J. 758, 75 (2012). 
[57] https://github.com/samodeo/Mop-c-GT.

[58] N. Itoh, S. Nozawa, and Y. Kohyama, Astrophys. J. 533, 588 (2000).

[59] M. Hilton et al. (ACT Collaboration), Astrophys. J. Suppl. Ser. 253, 3 (2021).

[60] S. Nozawa, N. Itoh, Y. Suda, and Y. Ohhata, Nuovo Cimento Soc. Ital. Fis. 121B, 487 (2006).

[61] S. Eales et al., Publ. Astron. Soc. Pac. 122, 499 (2010).

[62] D. Foreman-Mackey, D. W. Hogg, D. Lang, and J. Goodman, Publ. Astron. Soc. Pac. 125, 306 (2013).

[63] J. C. Hill, E. J. Baxter, A. Lidz, J. P. Greco, and B. Jain, Phys. Rev. D 97, 083501 (2018).

[64] A. J. S. Hamilton, P. Kumar, E. Lu, and A. Matthews, Astrophys. J. 374, L1 (1991).

[65] R. Nityananda and T. Padmanabhan, Mon. Not. R. Astron. Soc. 271, 976 (1994).
[66] R. Juszkiewicz, V. Springel, and R. Durrer, Astrophys. J. 518, L25 (1999).

[67] R. K. Sheth, A. Diaferio, L. Hui, and R. Scoccimarro, Mon. Not. R. Astron. Soc. 326, 463 (2001).

[68] S. Bhattacharya and A. Kosowsky, Phys. Rev. D 77, 083004 (2008).

[69] S. W. Henderson et al., J. Low Temp. Phys. 184, 772 (2016).

[70] G. J. Stacey et al., Proc. SPIE Int. Soc. Opt. Eng. 10700, 107001M (2018).

[71] Simons Observatory Collaboration, J. Cosmol. Astropart. Phys. 02 (2019) 056.

[72] M. Levi et al., Bull. Am. Astron. Soc. 51, 57 (2019).

[73] B. A. Benson et al., Proc. SPIE Int. Soc. Opt. Eng. 9153, 91531P (2014).

[74] K. N. Abazajian et al., Astropart. Phys. 63, 66 (2015). 